\title{
The Relation of Ethnic Identity to Self-Esteem and Academic Self- Concept of African American Students at a Predominantly White Institution
}

Drummond Russell Bush

Follow this and additional works at: https://researchrepository.wvu.edu/etd

\section{Recommended Citation}

Bush, Drummond Russell, "The Relation of Ethnic Identity to Self-Esteem and Academic Self-Concept of African American Students at a Predominantly White Institution" (2015). Graduate Theses, Dissertations, and Problem Reports. 5289.

https://researchrepository.wvu.edu/etd/5289

This Dissertation is protected by copyright and/or related rights. It has been brought to you by the The Research Repository @ WVU with permission from the rights-holder(s). You are free to use this Dissertation in any way that is permitted by the copyright and related rights legislation that applies to your use. For other uses you must obtain permission from the rights-holder(s) directly, unless additional rights are indicated by a Creative Commons license in the record and/ or on the work itself. This Dissertation has been accepted for inclusion in WVU Graduate Theses, Dissertations, and Problem Reports collection by an authorized administrator of The Research Repository @ WVU.

For more information, please contact researchrepository@mail.wvu.edu. 
The Relation of Ethnic Identity to Self-Esteem and Academic Self-Concept of African American Students at a Predominantly White Institution

Drummond Russell Bush, MA

Dissertation submitted to the College of Education and Human Services

at West Virginia University in partial fulfillment of the requirements

for the degree of

Doctor of Philosophy

in

Counseling Psychology

Jeffrey Daniels, $\mathrm{PhD}$, Chair Jennifer Taylor, $\mathrm{PhD}$, Committee Member George Mamboleo, PhD, Committee Member

Ed Jacobs, PhD, Committee Member John Blake, $\mathrm{PhD}$, Committee Member

Department of Counseling, Rehabilitation Counseling, and Counseling Psychology

Morgantown, West Virginia 2015

Keywords: African American, ethnic identity, self-esteem, academic self-concept

Copyright 2015 Drummond R. Bush 


\begin{abstract}
The Relation of Ethnic Identity to Self-Esteem and Academic Self-Concept of African American Students at a Predominantly White Institution

Drummond R. Bush

Social scientists have challenged professionals and educators alike to develop a better understanding of the influences a predominantly White environment has on the academic and interpersonal experiences of African American college students. This study explored the psychosocial impact of several factors, both dispositional (e.g., ethnic identity, self-esteem, and academic self-concept) and contextual (e.g., racial composition of the institution), for 91 African American college students enrolled at a predominantly White institution in the mid-Atlantic region of the United States. Pearson correlations indicated the other group orientation sub-factor of ethnic identity is positively correlated with self-esteem and academic self-concept. Multiple regression analyses indicated that grade point average is best predicted by academic selfconcept. Limitations of the present study, implications, and suggestions for future research are presented.
\end{abstract}




\section{ACKNOWLEDGMENTS}

Although dissertations are considered "independent projects", I could not have done this without the support of several important people along the way. First, the passion I have for culture and service is attributable to my loving mother. It is her love and support that has guided me in my pursuit of education and service to others. I am grateful to my wonderful family, as well. My parents' steadfast love and constant encouragement is remarkable. In subtle ways, they endeavor to change the lives of others for the better, and their humble, selfless approach has been my constant inspiration.

Within academia, countless teachers have inspired my love for learning. In high school, Dr. Joe Kuban always believed in me and strongly encouraged me to pursue higher learning. He instilled a passion for lifelong learning and helped cultivate my love for the social sciences. I am also deeply appreciative of the efforts of my doctoral chair, Dr. Jeffrey Daniels, for the numerous hours he has committed to helping me grow professionally, for all of the many edits, revisions, and IRB entanglements he helped navigate throughout the dissertation process. His thoughtful guidance, helpful feedback, and understanding were all critical to my success. At West Virginia University, I am very grateful for my professors, my clinical supervisors, and my doctoral committee for their valuable feedback and contributions towards my professional development.

Finally, I am grateful to the participants of this study. It takes trust and courage to respond to questions exploring ethnicity/race and education, and I appreciate their willingness to do so. I am also very appreciative of their generosity with their time and thoughtful responses. 


\section{TABLE OF CONTENTS}

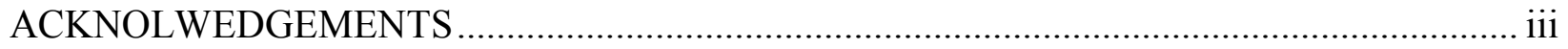

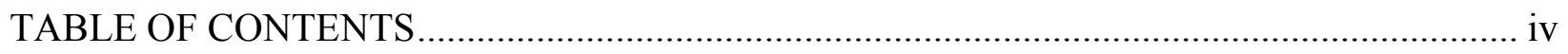

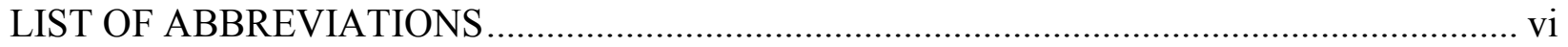

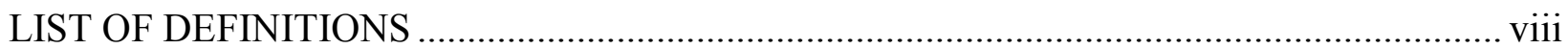

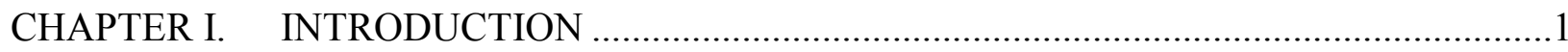

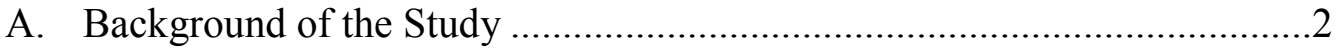

B. Statement of the Problem ...............................................................

C. Strategies for Success .................................................................... 8

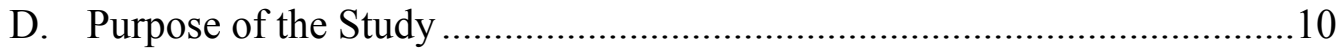

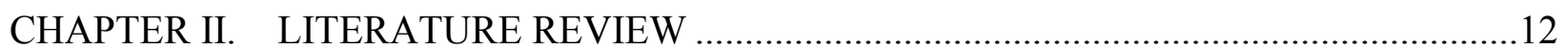

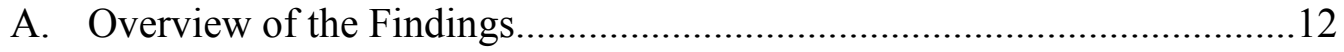

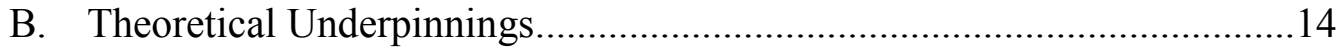

C. Models of Ethnic \& Racial Identity Development .................................19

D. Psychosocial Realities in Education ......................................................25

E. Self-Esteem of African American Students .........................................27

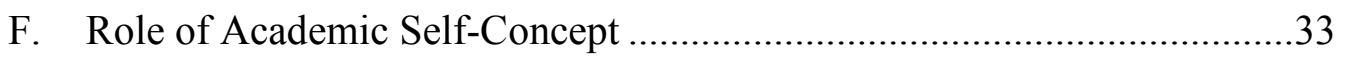

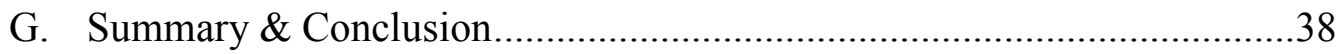

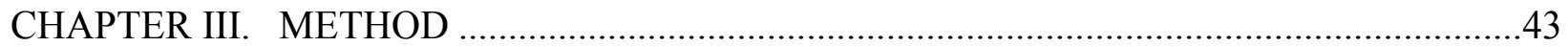

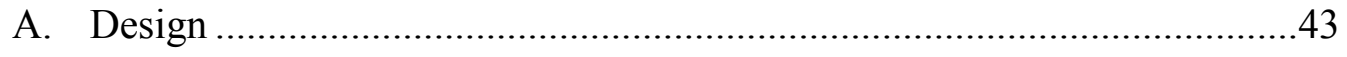

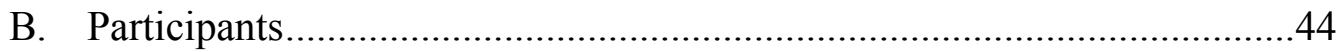

1. Sample size and power ................................................................45

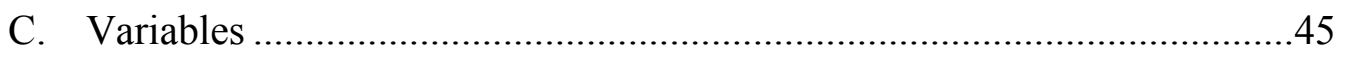

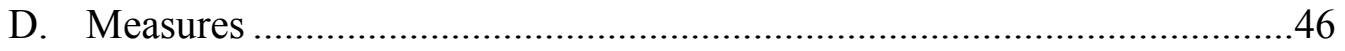

1. Multigroup Ethnic Identity Measure (MEIM).................................46

2. Rosenberg Self-Esteem Scale (RSES) ..........................................47

3. Academic Self-Concept Scale (ASCS) .........................................49

4. Marlowe-Crowne Social Desirability Scale: Form C (MC-C)...........52

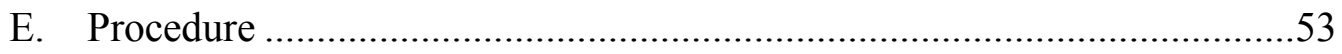

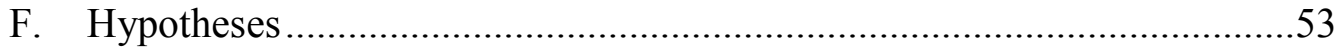

CHAPTER IV. RESULTS 


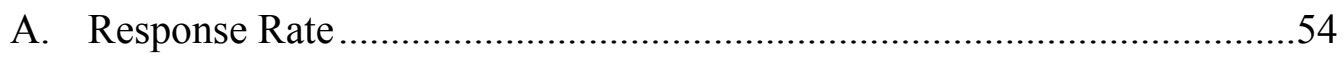

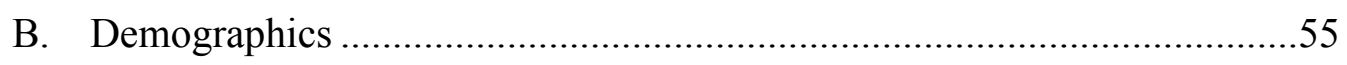

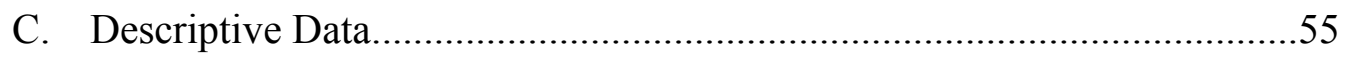

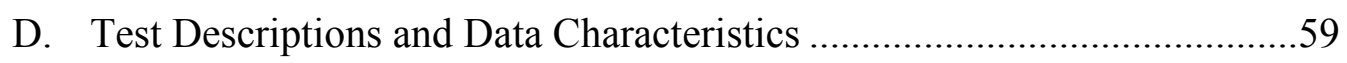

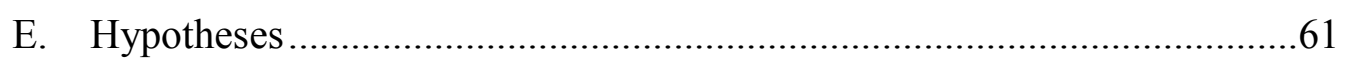

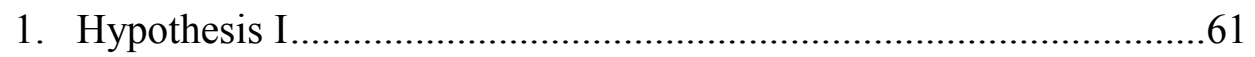

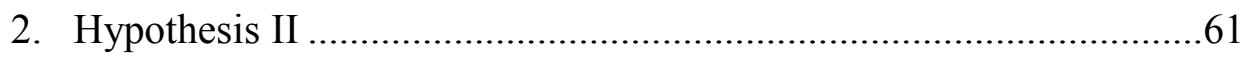

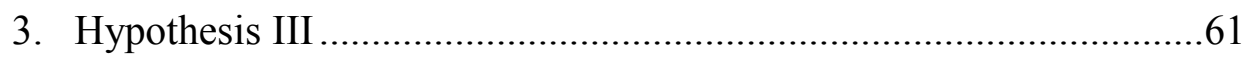

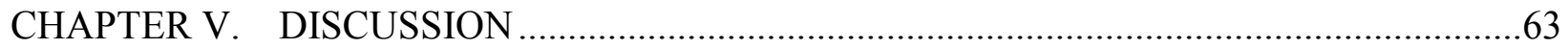

A. Multigroup Ethnic Identity Measure and ASCS, RSES, and GPA............63

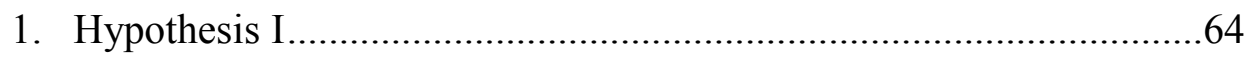

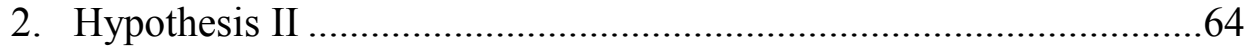

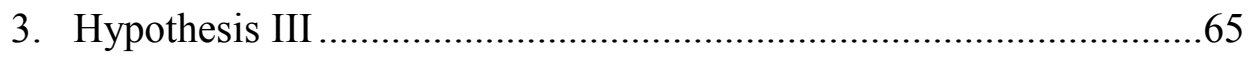

B. Comparisons Based on Institutional Context ........................................66

C. Limitations \& Strengths of the Present Study........................................67

D. Implications \& Suggestions for Future Research and Practice.................71

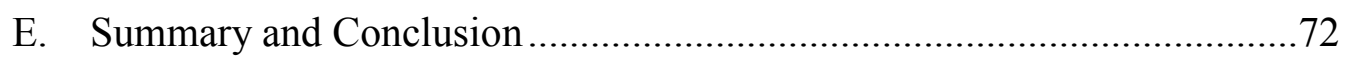

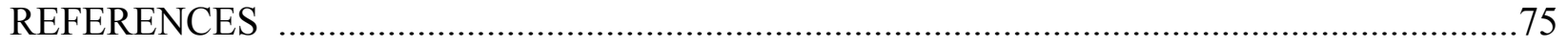

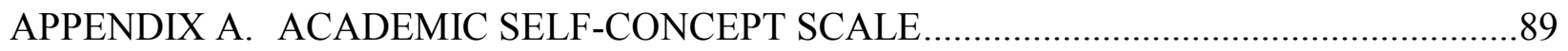

APPENDIX B. MULTIGROUP ETHNIC IDENTITY MEASURE ......................................91

APPENDIX C. ROSENBERG SELF-ESTEEM SCALE .................................................93

APPENDIX D. MARLOWE-CROWNE SOCIAL DESIRABILITY SCALE: FORM-C ..........94

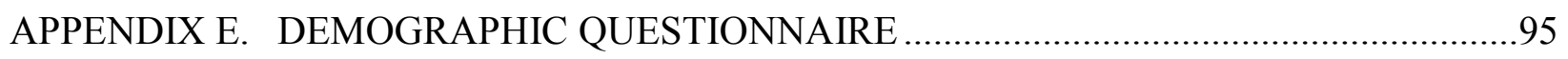

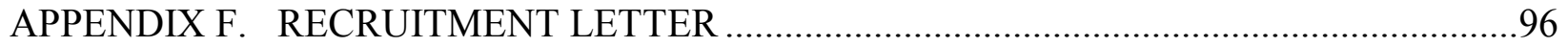

APPENDIX G. DEMOGRAPHIC SUMMARY OF PARTICIPANTS ...............................97 


\section{LIST OF ABBREVIATIONS}

$\begin{array}{ll}\text { ANOVA } & \text { Analysis of Variance } \\ \text { APA } & \text { American Psychological Association } \\ \text { ASCS } & \text { Academic Self-Concept Scale } \\ \beta & \text { Standardized Regression Coefficient } \\ \text { CIDC } & \text { Children of Immigrants Development in Context } \\ \text { EI } & \text { Ethnic Identity } \\ \text { GPA } & \text { Grade Point Average } \\ \text { HBCUs } & \text { Historically Black Colleges \& Universities } \\ \text { IQ } & \text { Intelligence Quotient } \\ \text { KSAs } & \text { Knowledge, Skills, \& Abilities } \\ \text { M } & \text { Mean } \\ \text { MC-C } & \text { Marlow Crowne: Short Form C } \\ \text { MSCDS } & \text { Marlow Crowne Social Desirability Scale } \\ \text { MCT } & \text { Multicultural Counseling Theory } \\ \text { MEIM } & \text { Multigroup Ethnic Identity Measure } \\ \text { N } & \text { Number of participants } \\ \text { NCES } & \text { National Council on Education Statistics } \\ \text { OGO } & \text { Other Group Orientation } \\ \text { POI } & \text { P-value } \\ \text { PWI } & \text { Predominantly White Institution } \\ \text { Correlation Coefficient }\end{array}$


Running head: THE RELATION OF ETHNIC IDENTITY

RIAS Racial Identity Attitudes Scale

RSES Rosenberg Self-Esteem Scale

SAT Scholastic Aptitude Test

SD Standard Deviation

SIT Social Identity Theory

SPSS Statistical Package for the Social Sciences

t Coefficient Divided by Standard Error 


\section{LIST OF DEFINITIONS}

Academic Disidentification: a progressive state of being in which the general self-concept becomes increasingly less associated with academic performance the longer one stays in school (Steele, 1992).

Academic Self-Concept: a student's views about his/her academic ability as compared to other students (Cokley, 2000).

Bonding: an engagement that promotes and sustains connectedness; it is often the result of progressive unification rather than compulsory connection (Cross \& Strauss, 1998).

Bridging: an organized and focused effort that promotes interaction with out-group/dominant group members (Cross \& Strauss, 1998).

Buffer: anything that provides psychological protection against racist situations and facilitates stigma management (Cross \& Strauss, 1998).

Code Switching: a mixture of attitudinal, behavioral, and conversational modifications done to fit societal expectations (Koch, Gross, \& Kolts, 2001).

Ethnic Identity: one's subjective sense of ethnic group membership, which involves selflabeling, sense of belonging, preference for the group, positive evaluation of the ethnic group, ethnic knowledge, and involvement in ethnic group activities (Cokley, 2007).

Ethnicity: the combination of cultural characteristics (e.g., language, religion, vocational choice, and family constellation) which contribute to one's identity (Fischer \& Moradi, 2001)

Multiculturalism: a social and intellectual movement that encourages the valuing of diversity as a core belief and maintains that all cultural groups be treated equally and with respect (Fowers \& Davidov, 2006). 
Other Group Orientation: an assessment of attitudes toward, and interactions with, ethnic groups other than one's own (Phinney, 1992).

Out-group Comfort: one's degree of social comfort with out-group members; thought to serve as a resource allowing social ease with dominant group members (Cole \& Yip, 2008).

Psychological Disengagement: a self-defense strategy used to protect one's self-esteem from academic performance so that self-esteem is not contingent upon one's successes or failures in that domain (Steele, 1997).

Race: a social construct primarily based on physical features rooted in continental origin, and the most common features include: skin pigmentation, head form, nasal index, lip form, and distribution/texture of hair (Clark, Clark, \& Williams, 1999).

Racial Centrality: the extent to which one maintains group pride and positive beliefs about society’s views of African Americans (Chavous et al., 2003).

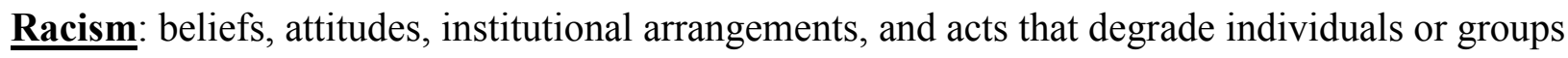
because of phenotypic characteristics or ethnic group affiliation (Clark, Anderson, Clark, \& Williams, 1999).

Self-Esteem: the positive or negative feelings one has about oneself (Richardson, Ratner, \& Zumbo, 2009).

Social Desirability: the effort to appear overly moral, honorable, and virtuous by denying common yet undesirable traits, and/or exaggerating uncommon yet desirable traits (Crowne \& Marlowe, 1960).

Stereotype Threat: a psychosocial process wherein African American students begin to fear their behavior (i.e., lower academic performance) will confirm the stereotypes associated with their group (Steele, 1997). 


\section{CHAPTER I}

\section{INTRODUCTION}

In the modern collegiate landscape, nearly $90 \%$ of the African American students enrolled at degree granting institutions attend predominantly White institutions (PWIs) (National Center for Educational Statistics, 2014). The opportunity for African Americans to attend PWIs grew exponentially with the desegregation of public schools following the landmark Brown v. the Board of Education of Topeka, Kansas (1954) decision. This judgment led to the widespread abolishment of restrictions placed on minority students' admission to PWIs and granted African Americans broader access to degree granting institutions. Desegregation in academia provided hope for many African Americans previously denied access, and seemed to suggest that African Americans would begin to matriculate quickly and graduate from PWIs at the same rate, or greater, than they had achieved at Historically Black Colleges and Universities (HBCUs) (Arminio et al., 2000; Toldson \& Cooper, 2014).

By 1974, approximately three-fourths of the African American college student population was attending a PWI (Palmer \& Young, 2009). According to the NCES Digest of Education Statistics (2014), African Americans contribute to approximately 15\% of the total college student population; yet, only about $10 \%$ of the African American students who enrolled for the Fall, 2013 semester (or 311,671 out of the 2,790,255) selected an HBCU. However, despite the increased enrollment of African Americans at PWIs, HBCUs are showing greater improvements in African American student graduation rates (Toldson \& Cooper, 2014).

For example, in 1998 the African American student graduation rate at Howard University, an HBCU in Washington, D.C., was approximately 47\%. By 2012, the graduation rate for African American students at Howard had increased to 63\%, as compared to the 34\% 
national graduate rate of for all other African American college students (e.g., those attending PWIs). In 2012, seven HBCUs reported a graduation rate of $50 \%$ or better (Toldson \& Cooper, 2014).

To assist in the understanding of racial/ethnic differences in academia, a discussion of the dispositional attributes and environmental variables found to influence the college experience for African American students is offered. Secondarily, an overview of the research focused on the impact of race and ethnicity on identity formation processes, particularly for African American college students at PWIs, is provided as a contextual frame for the reader.

\section{Background of the Study}

Mickelson (1990) stated that African American students generally earn lower grades, drop out more often, and achieve less education than do White students. For African American students at PWIs, researchers have found that the predominantly White environment has a greater likelihood to be experienced as prejudicial; thereby suggesting PWIs could potentially be experienced as more distressing when compared to an HBCU setting (Sue et al., 2007). This is presumably due, in part, to the presence of seemingly unavoidable social realities within a PWI environment, such as racism (overt and/or covert in nature) and other sordid stressors that might negatively influence a healthy and productive lifestyle (Burkard, Medler, \& Boticki, 2001; Sue et al., 2007; Thompson, Anderson, \& Bakeman, 2000). In addition, African American students at PWIs interact with Whites on a regular basis; therefore, they are vulnerable to the cumulative effects of racism and prejudice (Cole \& Yip, 2008; Morin, 2001; Sue et al., 2007).

In an oft cited publication titled "Blacks in College", an African American student author named Jacqueline Fleming (1984) advised readers that PWIs are commonly experienced as unaccepting environments, consequently obstructing the intellectual growth of many African 
American students. She suggested that, despite the gains made since the civil rights movement, anti-Black racism continues to be prevalent on college campuses (Branscombe, Schmitt, \& Harvey, 1999; Chavous, 2000; Cole \& Yip, 2008). Although modern society largely espouses the belief that anti-Black racism in the United States has declined over the past 50 years, a recent literature review shows "attitudes toward Affirmative Action have stayed fairly constant since 1970, with $20 \%$ or fewer of Whites approving of such programs across time" (Arriola \& Cole, 2001, p. 2465).

What is more, regardless of the heterogeneity existent within any African American college student population, racial stereotypes have the potential to affect the academic experience for all African American students. For example, Fries-Britt and Griffin (2007) found that student based assumptions of being an Affirmative Action attendee led African American students to: 1) Monitor their behavior for appropriateness, fit; 2) Develop concerns about being a "token" member of his/her ethnic group; 3) Experience added pressure to balance bicultural tensions; and 4) Succumb to pressure to prove their worthiness to attend school.

Social scientists overwhelmingly agree that the history of racial discrimination in the United States has left a lasting residue, even in a society that overtly detests discrimination (Smedley \& Smedley, 2005). Likewise, discrimination based on race has been noticeably expressed with little or no societal repercussions throughout most of the $20^{\text {th }}$ century (Burkard et al., 2001). Considering this, it appears likely that an experience with discrimination (particularly for African American students at PWIs) is somewhat of an expected reality. More importantly, these potential encounters with discriminatory experiences could have a significant impact on the attainment of personal and professional goals. 
In order to develop an understanding of the numerous individual identities that contribute to the African American college student experience, one must consider the influence of factors such as race and ethnicity, especially as they relate to issues of self-appraisal and academic performance. It appears that race is a fairly recent construct; and, the construct emerged well after population groups from different continents encountered one another (Smedley \& Smedley, 2005). Race is primarily based on physical features rooted in continental origin, and the most common features include: skin pigmentation, head form, nasal index, lip form (and color), and distribution/texture of hair (Clark, Clark, \& Williams, 1999). Interestingly, with regards to physical similarities, there is often more within, than between group variance (Cardemil \& Battle, 2003; Clark et al., 1999).

Ethnicity, as opposed to race, is a more broadly defined construct; therefore, the attention of ethnic identity researchers (as opposed to racial identity researchers) is generally more nuanced. Ethnic identity research often focuses on the acquisition and maintenance of cultural characteristics such as language, or other forms of individual cultural expression (Worrell \& Gardner-Kitt, 2006). The variables which contribute to one's culture (e.g., religion, education, vocational choice, family constellation, etc.) are also viewed as components of an individual's ethnic identity (Fischer \& Moradi, 2001; Seidner, 1982). People appear to operate from their own unique perspective with regards to ethnic identity, and the stages of ethnic identity are best viewed as fluid rather than crystallized in nature (Phinney, 1996).

African American psychologist William Cross (1971), a pioneer in the study of Black racial identity theory, suggested that Black racial identity development followed a discrete attitudinal model, and people could therefore be placed in distinctive categories. However, in adapting the Cross (1971) model to study counseling variables, Parham and Helms (1981) argued 
that attempts to simply categorize African Americans into fixed stages of Black racial identity were misleading. They suggested that placing people in a fixed stage of racial identity ignored the presence of simultaneous, and potentially more fluid, developmental perspectives.

In 1981, Parham and Helms challenged the static nature of Cross' (1971) Black racial identity model; and their contribution has significantly impacted our understanding of racial and ethnic identity development. Ultimately, they maintained that African Americans progressed through four psychological stages as they evolved from a self-perception where Black racial identity is degraded to a self-perception in which they are secure with being Black. They termed these stages Preencounter, Encounter, Immersion-Emersion, and Internalization.

A few years later, based largely on pre-existent models of racial identity, psychologist Jean Phinney established a model of ethnic identity development. Ethnic identity was intended to be more idiographic and continuum based than previously identified racial identity models (Phinney, 1989). Phinney described ethnic identity as a dynamic construct that changes over time and context and varies across individuals (1996). Phinney developed a three stage model of ethnic identity development in 1989 , which was based on her research with minority adolescents wherein she utilized other ego identity and racial identity models; she tended to borrow heavily from identity theorists James Marcia (1980) and Erik Erickson (1968). Phinney's research indicated that minority adolescents seemed to share traits, similar enough to one another, that they could be grouped into one of three stages of ethnic identity (i.e., the unexamined stage, search/moratorium stage, and achieved stage) (Phinney, 1989; 1992; 1996).

However, Phinney strongly maintained that those studying the construct of ethnic identity should understand the psychological aspects of ethnicity first, in order to avoid placing too much emphasis on the group label itself (1992). Thus, the three psychological aspects of ethnicity 
identified by Phinney are culture, ethnic identity, and minority status. The first of these aspects, culture, refers to an adherence to values, beliefs, behaviors and norms associated with one's cultural group. The second psychological aspect, ethnic identity, refers to the extent to which one identifies with one's ethnic group. It is the meaning, strength, and salience of one's ethnicity. Lastly, the third psychological aspect, minority status, refers to the extent to which one has the differential experiences and attitudes that are associated with minority status. These attitudes are often based on being a part of a minority group that is frequently the target of racist behaviors and prejudicial attitudes (Phinney, 1996).

Ethnic identity formation has to do with developing an understanding and acceptance of one's own group in the face of lower status and prestige in a society that often perpetuates stereotypes and racism (Phinney, 1996). Decades of extant literature reveal that ethnic identity is a complex multi-factorial concept; therefore, it is strongly discouraged to view ethnic identity development as a stagnant process, or one-dimensional in nature. People's attitudes and behaviors change over time, and these changes can have important implications on the ways in which individuals live their lives, interact with people from other groups, and view society as a whole.

In order to understand the complexity associated with the African American college student experience at PWIs, one must have a foundational understanding of the concept of racism. Racism is defined as "beliefs, attitudes, institutional arrangements, and acts that denigrate individuals or groups because of phenotypic characteristics or ethnic group affiliation"

(Clark, Anderson, Clark, \& Williams, 1999, p. 806). The perception of racism usually results in some type of psychological and physiological stress response for those who experience it. Therefore, the need for African American students at PWIs to navigate between cultures may 
unknowingly predispose them to tension and stress in the environment; thereby, potentially limiting their overall performance and general satisfaction within predominantly White settings.

\section{Statement of the Problem}

The available research suggests that perceptions of both in-group and out-group racism have persisted, and they continue to exert a significant effect on the well-being of many African Americans (Clark et al., 1999; Cole \& Yip, 2008; Parham \& Helms, 1985). To the extent that African Americans may, for historical reasons or personal experience, be particularly sensitive to signs of rejection, dislike, or discrimination, they are likely to weigh the negative signals more heavily than the positive overt behavior (Sellers \& Shelton, 2003; Shelton, 2000). This increased sensitivity is cause for concern because negative signals are often covert in nature, thus rendering them more difficult for out-group members to detect and change (Sue et al., 2007).

Researchers studying the development of college students have suggested that in addition to the challenges of college transition, African American students also confront the task of ethnic identity development and negotiating interactions within the dominant culture (Cole \& Yip, 2008). The psychosocial component of individual ethnic identification involves an acute awareness of boundaries between members of different ethnic groups. These boundaries are both explicit and implicit, as they are imposed internally by members of the group, and externally by society at large (Arroyo \& Zigler, 1995).

Psychologists Cole and Yip (2008) conducted a two-year longitudinal study of African American college freshmen $(n=143)$ at a PWI in the northeast. This study investigated whether African American students' social comfort with Whites predicted outcomes related to academics and mental health. Among their findings, Cole and Yip (2008) demonstrated that an African 
American student who identified as having experienced prejudice from a White professor endorsed less social comfort with Whites and held a comparatively lower grade point average.

Similarly focused researchers (i.e., Arroyo \& Zigler, 1995; Mickelson, 1990; Ogbu, 1978; Steele, 1992) have identified other contributing factors (e.g., disproportion in the social and educational systems, academic de-identification, etc.) that are believed to have led some African Americans to develop a perception of college as futile. Therefore, it is incumbent upon teachers and researchers alike to work toward alleviating these barriers and to develop/implement approaches that are mindful of the unique experiences of African American students, especially in predominantly White environments.

\section{Strategies for Success}

It is assumed that the African American students who effectively manage experiences with prejudice and discrimination have developed a number of adaptive strategies to aid in their negotiation of racially imbalanced social environments (Utsey, Ponterotto, Reynolds, \& Cancelli, 2000). Cross and Strauss (1998) identified several healthy aspects of African American identity and function. Their research focused on the individual pursuit of goals related to self-development and the eventual attainment of ethnic/racial identity end-states. Although identity strategies primarily reflect the ways in which individuals negotiate self and context (i.e., goal-directed behavior), identity functions, on the other hand, are focused on the purely selfrelevant goals individuals try to achieve en-route to self-actualization (Cross \& Strauss, 1998).

For example, the act of "buffering" is assumed to be a healthy identity function associated with African American identity. Buffering is assumed to provide psychological protection against racist situations and facilitates stigma management (i.e., it acts as a filter against racism and aids in the differentiation between stigma-tainted vs. race-neutral 
information) (Cross \& Strauss, 1998). The following are some examples of buffering that have been identified to aid in coping with racism: belonging to a higher social class, living in racially integrated environments, a strong sense of familial support, and possessing less visible signs of racial difference (e.g., lighter skin pigment) (Cross \& Strauss, 1998; Utsey et al., 2000).

Cross and Strauss (1998) identified several other characteristically adaptive identity functions for African Americans; among them are the notions of bonding, bridging, and code switching. Bonding and bridging are similar with regards to their appearance and role.

Bonding is the promotion and sustainment of connectedness, which is distinguishable from bridging, as bonding is more the result of progressive unification rather than compulsory connection. Examples of bonding might include activities like playing on a sports team, being involved in a band, and engaging with a university club or campus organization.

Conversely, bridging activities typically reflect more of an organized and focused effort to help build connections with out-group members (e.g., campus events during "Black History Month”); therefore, bridging occurs more so in response to observed racial and ethnic differences. Typically, the bridging experiences for African Americans at PWIs are institutionally supported and are based on the notion of promoting healthy understanding and integration amongst students from diverse cultural backgrounds (Koch, Gross, \& Kolts, 2001).

Code switching refers to the conscious process of turning off, or switching on, one's tendency to present self in a "Black" way (Cross \& Strauss, 1998). Although this concept is generally used in journals of pragmatics, where it refers to bilingual individuals alternating language usage, code switching can also be viewed as a combination of attitudinal, behavioral, and conversational modifications done to fit societal expectations (Koch, Gross, \& Kolts, 2001). Cross and Strauss conceptualized code switching as an identity function; however, it could also 
be understood as a strategy, as it is a means by which individuals make an effort to convey a more appropriate identity in a given context.

Code switching appears to be particularly adaptive when an organization or group of people (e.g., school, workplace, neighborhood, or restaurant) displays evidence of discomfort with explicit expressions of difference. In other words, the social benefit of code switching is based on the assumption that if an individual speaks and acts similar to the positively viewed majority, or in-group, then the person will likely be viewed as possessing positive attributes; conversely, individuals who speak and act differently from the in-group may be perceived as possessing undesirable traits (Koch, Gross, \& Kolts, 2001). Interestingly, the drive to incorporate code switching behaviors is influenced by both majority and minority cultures (Cross \& Strauss, 1998); and, it appears that in order to maintain cultural ties and simultaneously advance in a

predominantly White environment, code switching between minority and majority language and behaviors may serve as an adaptive function.

\section{Purpose of the Study}

In summary, there are various social, political, cultural, and other realities that influence the African American college student experience at PWIs. Now, while some of the experiences accompanying PWI environments may potentially contribute negatively to the development of African American students, PWIs also provide an increased opportunity for adaptive crosscultural encounters and positive developmental experiences that can have a significantly positive effect on personal growth and awareness.

Considering the fact that African Americans currently attend PWIs at a rate higher than any other numerical minority (NCES, 2014), it is advisable that helping professionals and educators alike do their part to attune to the needs of African American students. Based on the 
literature related to African American ethnic identity development/function, selfesteem, and academic performance, the following research questions will be examined:

- Are the self-esteem and academic self-concept of African American college students at a PWI related to individual variations in ethnic identity?

- What is the relationship between ethnic identity, self-esteem, academic self-concept, and grade point average (GPA); and, what is the best predictor model for GPA? 


\section{CHAPTER II}

\section{LITERATURE REVIEW}

The literature review below is comprised of four parts. First, to frame the argument, an overview of the literature exploring the impact of racial/ethnic identity on healthy development and functioning will be presented. Second, an introduction to Social Identity Theory (SIT) and Multicultural Counseling Theory (MCT) will be provided in an effort to underpin the developmental view of racial and ethnic identity. A clarification of the respective differences between SIT and MCT is then offered, along with a general exposure to ethnic and racial identity models. Third, a comprehensive review of the extant research on the psychosocial factors relative to identity development processes for African American college students will be presented. The role of academic self-concept for African American students will then be discussed, particularly as it relates to self-esteem and ethnic identity. To conclude, a description of Witherspoon, Speight, and Thomas' (1997) study of racial identity, self-esteem, and academic self-concept among African American high school students will be provided, in an effort to demonstrate the value of conducting a modified replication and extension of this study. Finally, inferences based on this literature review are asserted and a study based on this query is then proposed.

\section{Overview of the Findings}

The primary purpose of this literature review is to illuminate the psychological impact of the sociocultural realities experienced by African American students in the current educational landscape. That said, research on ethnic identity continues to be a popular topic in counseling psychology, as evidenced by the increasing number of theses and dissertations devoted to the topic (Cokley, 2005). Ethnic identity can be broadly defined as one's subjective sense of ethnic 
group membership, which involves self-labeling, sense of belonging, preference for the group, positive evaluation of the ethnic group, ethnic knowledge, and involvement in ethnic group activities (Cokley, 2007). The African American community, as an "ethnic group", has a wellestablished tradition of valuing higher learning and education (Fordham \& Ogbu, 1986; Ogbu, 1978); likewise, African Americans' integration and maintenance of these values (i.e., higher learning and education) remains evident in the current landscape. For African American students, individual motivation to pursue academic goals has been linked to the awareness of African Americans' past, and current, struggles for educational access and opportunity (Chavous et al., 2003; Fordham \& Ogbu, 1986; Ogbu, 1978).

In 2000, Thompson, Anderson, and Bakeman developed a study to determine if racial identity mediated the relationship between racial socialization and acculturative stress in African American college students $(n=84)$. They discovered that racial socialization was positively correlated with higher levels of racial identity (i.e., immersion and internalization attitudes), and that racial identity was generally associated with the experience of acculturative stress. Furthermore, study participants who endorsed primarily pre-encounter attitudes were found to be at an increased risk for substance abuse (i.e., Townsend \& Belgrave, 2000), as well as academic difficulties (Thompson et al., 2000)

Such a potentially negative reality may likely be influenced by the social discomfort and subsequent disengagement predominantly White environments might foster. For example, social scientists have demonstrated that the African American students who endorse an achieved ethnic identity are typically found to have more comfortable and adaptive social interactions, which translates to healthier overall functioning (Allen, 1992; Cokley, 2002; Cokley, 2005; Cole \& Yip, 2008). Moreover, individuals at later stages of ethnic identity development have a tendency 
to engage their environment in a more frequent and assertive manner. Specific behaviors such as enrolling in African American Studies courses, having an African American best friend, and/or having more general social contact with African Americans have been shown to be conducive to the individual growth experience as well (Rowley, Sellers, Chavous, \& Smith, 1998). More importantly, these findings suggest that African American students at PWIs benefit from contact and involvement, especially with other African Americans, as this seems to be advantageous to the development of an achieved ethnic identity.

An African American student attending a PWI, presumably with a more achieved ethnic identity, might utilize his/her ethnic identity as a buffer amidst the potential stressors of the environment. Furthermore, out-group comfort, or social comfort with Whites, may serve as an additional resource allowing social ease with dominant group members (Cole \& Yip, 2008). Consistent with the critique of deficit models in the psychology of African Americans, out-group comfort is viewed as a personal strength, rather than as a disavowal of one's own culture or an internalization of oppression (Smith, 2006). However, there are competing theoretical perspectives which attempt to explain the individual and group processes involved in adaptively orienting oneself to the out-group and developing an achieved ethnic identity.

\section{Theoretical Underpinnings}

Social Identity Theory (SIT) and the emergent Multicultural Counseling Theory (MCT) provide two possible explanations for the development of group and individual identification amongst African American college students. Social Identity Theory proposes that identification with one's group is linked to a belief in in-group superiority which, in turn, satisfies the need for positive self-esteem (Negy et al., 2003). It is assumed that in-group bias is evident from an early age and continues throughout the lifespan (Yee \& Brown, 1992). According to Verkuyten 
(2005), group categorization, social comparison, and the need for positive differentiation are the key psychological mechanisms used for understanding intergroup relations in SIT.

Group members are anticipated to react toward other groups out of a need to distinguish their own group positively. This is based on the assumption that group members derive their social identity from membership in social groups; likewise, it is then presumed that individuals favor their own culture to be socially recognized, accepted, and valued. Thus, the experience of sociocultural recognition and valuing for African American students at PWIs could contribute to a more meaningful and positive social identity. However, it appears that SIT is more a theory of in-group bias, rather than a philosophy of out-group derogation. Beyond the assumed preference of one's own ethnic group over others, SIT explains little about the attitudes ingroup members maintain toward out-group members (Phinney, Jacoby, \& Silva, 2007). Comparatively, MCT allows for an enriched and hopefully more adaptive perspective toward intergroup relations. It emphasizes the malleability of group identity and intergroup attitudes across the lifespan (Ivey, D’Andrea, Ivey, \& Morgan, 2002; Sue, 2004; Verkuyten, 2005).

The Multicultural Theory hypothesis proposes that affirmation toward one's ethnic group leads to a positive ethnic identity and higher levels of acceptance toward ethnic out-groups (Sue, Ivey, Pedersen, 1996). In addition, multiculturalism has been described as both a social and intellectual movement that encourages the valuing of diversity as a core belief and maintains that all cultural groups be treated equally and with respect (Fowers \& Davidov, 2006). Yet, multiculturalism is defined differently and takes altered forms in schools, organizations, and countries, for example; however, common arguments underlie these differences. In general, multiculturalism tries to foster understanding and appreciation of ethnic diversity by acknowledging and respecting minority group identities and cultures. 
According to the American Psychological Association (APA), "environments which seek to foster the establishment and maintenance of 'multiculturalism' are expected to lead to the development of more positive and secure ethnic identities" (2003, p. 386). Likewise, the proposed tenants of MCT suggest multiculturalism leads to increased openness toward, and acceptance of, the other (Fowers \& Davidov, 2006; Sue, 2004). Currently, there is little empirical evidence for the predicted effects of multicultural focused interventions, and recent research indicates it's unlikely that the effects of multiculturalism are similar for ethnic majority and minority groups (Cokley, 2007; Sue, 2004; Verkuyten, 2005).

Verkuyten (2005) examined the impact of multicultural ideology on group identification and ethnic group evaluations among ethnic minority and majority group members. His focus was on social identity processes, and he found that, in general, members of an ethnic minority group are more likely to endorse multiculturalism than are members of an ethnic majority group.

Moreover, members of the majority group reported less positive out-group evaluations than the minority group members, as well as a lesser degree of in-group identification. Consistent with SIT, the effects of ethnic group identification and group evaluation were moderated by individual differences in the endorsement of multiculturalism as an interethnic ideology. In other words, multiculturalism provides a justification for identity affirmation and cultural maintenance for ethnic minority groups, but not necessarily for majority groups.

The positive relationship between in-group preference and out-group prejudice, which is assumed in SIT, conflicts with both developmental theory and MCT (Phinney, Ferguson, \& Tate, 1997; Sue, 2004). Multicultural Theory tries to foster an understanding and appreciation of ethnic diversity by acknowledging and respecting minority group identities and cultures, postulating that individuals with a positive sense of their own culture will have positive attitudes 
toward other groups, as well as enhanced self-esteem (Ivey at al., 2002; Negy et al., 2003; Sue, 2004). Similarly, the developmental view holds that a more secure ethnic identity should be associated with greater acceptance of out-groups (Phinney et al., 2007).

Even if one adheres to the central tenants of MCT and the developmental view, they must also possess the willingness to acquire the necessary knowledge, skills, and abilities (KSAs) to interact positively and effectively with ethnically diverse populations. The developmental nature of MCT assumes that the acquisition of individual KSAs increases throughout one's life and has positive effects on both ethnic group identification and intergroup relations. In 2003, the APA introduced a set of six multicultural guidelines designed to help psychologists increase their knowledge and skills in multicultural education, training, research, practice, and organizational change.

In recent years, efforts focused on increasing multiculturalism have been progressively more recommended as an effective intervention at societal and local levels, yet very little is known about the specific ingredients that influence positive outcomes (Lockett \& Harrell, 2003). It is assumed that positive multicultural interactions, cross-cultural relationships, and personal adjustments result from a more developed ethnic identity (Phinney et al., 2007). Therefore, from a multicultural perspective, efforts to strengthen ethnic identity are generally recognized as ideal and are encouraged in the developmental process of all people.

A developmental perspective on intergroup relations offers a model of positive intergroup attitudes (Phinney et al., 2007). As opposed to SIT, which fails to attend to changes over time, a developmental approach to intergroup relations emphasizes that both group identity and intergroup attitudes undergo changes with age. Beginning at a young age, ethnic identity plays an integral part in many aspects of development, particularly among adolescents of color in the 
United States (Phinney, Ferguson, \& Tate, 1997). More specifically, for African Americans, an important aspect in conceptualizing their individual ethnic identities involves understanding the attitudes they have toward other ethnic and cultural groups (Cokley, 2005).

Phinney, Cantu, and Kurtz (1997) argued that a more secure ethnic identity is the result of a developmental process that typically takes place during adolescence and young adulthood, leading to confidence in one's own group membership and greater openness to other groups. As school-aged children form in-group and out-group social preferences and biases, including those based on ethnicity and race, the strength of pride and salience a child feels about his or her ethnicity plays an important role in developing inter-ethnic group social preferences (Marks et al., 2007).

The Children of Immigrants Development in Context Study (CIDC) conducted by Marks et al. (2007) demonstrated that ethnic identity increased with age and predicted positive in-group attitudes which, in turn, contributed to more positive out-group attitudes. This study explored the ethnic identity and group social preferences in middle childhood (ages 6-12) of Cambodian, Dominican, and Portuguese children who immigrated to the United States. The degree to which each child endorsed components of ethnic identity exploration, pride, and salience were related to out-group social preferences. Older children in this study generally reported more positive social desirability for children of other ethnic groups and had greater amounts of in-group pride and ethnic identity exploration. This finding supports previous research depicting that salience and strength of ethnic identity are important to social group preferences and generally vary throughout the lifespan (Verkuyten, 2005). 


\section{Models of Ethnic \& Racial Identity Development}

Among ethnic minorities in particular, researchers have discovered that a welldeveloped ethnic identity seems to serve as a secure position which allows individuals to be more open and accepting of people from other ethnic groups. By reducing feelings of threat, a strong ethnic identity may also allow ones natural tendency toward novelty to be a stimulus for initiating contact with people who are different (Phinney, Jacoby, \& Silva, 2007). Although the extant literature suggests that ethnic identity is a salient construct beginning in childhood, due to the emergent nature of the construct, it often becomes increasingly more complex and significant as one grows older (Cokley, 2007).

Both conceptual and empirical writings acknowledge that ethnic identity is a dynamic construct that changes over time and context, and varies across individuals. An achieved identity is accomplished only after individual exploration and commitments have occurred in a variety of domains (e.g., ideology, occupation, and lifestyle); these commitments then serve as a guide to future choices (Phinney, 1996). College is often the time in which individuals openly explore their career options and lifestyle choices; thus it appears that ethnic identity would be particularly relevant during the collegiate experience. Furthermore, a positive group identity could indeed function as a protective factor for many African American students attending PWIs.

In 1989, Phinney evaluated stages of ethnic identity development through interviews with 14 Asian-American, 25 Black, 25 Hispanic, and 27 White 10th graders (aged 15-17 years-old.), all US-born. All subjects were also given questionnaire measures of ego identity and psychological adjustment. Participants of color were coded as being in 1 of 3 identity stages; however, White subjects could not be reliably coded. Among the participants of color, about one-half had not explored their ethnicity (diffusion/foreclosure); about one-quarter were involved 
in exploration (moratorium); and about one-quarter had explored and were committed to an ethnic identity (achieved). Participants who endorsed an achieved ethnic identity had the highest scores on ego identity and psychological adjustment.

A few years later, Phinney (1992) endeavored to develop a questionnaire measure of ethnic identity based on the elements of ethnic identity that were common across groups. The relationship of ethnic identity to various demographic variables and to self-esteem was also examined. The questionnaire was administered to 134 Asian American, 131 African American, 89 Hispanic, 12 White, and 41 mixed background high school students (aged 14-29 years-old) and 58 Hispanic, 35 Asian, 23 White, 11 Black, 1 American Indian, and 8 mixed background college students (aged 18-34 years-old). Reliability, assessed by Cronbach's alpha, was .81 for the high school sample and .90 for the college sample. Upon demonstration of the questionnaire's reliability, the measure began to be used by researchers as a tool to examine similarities and differences in ethnic identity, as well as its correlates, among individuals from different ethnic groups.

As empirically supported research on ethnic identity began to mount, Phinney (1996) expanded the three stage model and provided significant additional knowledge to the understanding of ethnic identity. She emphasized the importance of exploring culture-specific factors, as well as universal factors, when attempting to understand ethnic identity. Therefore, an examination of African American culture-specific factors merits further investigation.

African American psychologists have long argued that an understanding of African American identity must focus on an ethnic and cultural identity (often rooted in an Afrocentric worldview paradigm), which critically examines and affirms African cultural values as forming the foundation of African American identity and culture (Cokley, 2005; Cross, 1971; Fordham \& 
Ogbu, 1986). Afrocentric values include an emphasis on spiritualism (i.e., belief in spirit as the basis of existence of everything), collectivism (i.e., giving priority to the goals of the family and ethnic group), communalism (i.e., emphasis on human relationships, recognizing every community member's value and uniqueness), and a belief in self-knowledge as the basis of all knowledge (Myers, 1988).

In 1971, a pioneering African American social psychologist named William Cross created the "Theory of Black Racial Identity Development." Cross' (1971) theory has been foundational to the subsequent development of numerous models of racial and ethnic identity over the past 40years (Cokley, 2007; Helms, 1990; Parham \& Helms, 1981; Phinney, 1992; Phinney, 1996; Sellers et al., 1997; Sellers et al., 1998). Cross expanded his theory in 1991, and created a five stage model to capture the African American culture-specific factors related to identity. The first stage of the model is pre-encounter, wherein attitudes fall along a continuum and range from race being an insignificant part of personal identity (race neutrality) to race being devalued by the individual to the point of self-hatred (race negative). The second stage is the encounter stage, in which attitudes about race are directly or indirectly challenged by some personal or social event that provides a means for the individual to transform his or her existing views of identity. Immersion/emersion is the third stage, and an individual's previously held racial attitudes are given up in an attempt to become more involved in his or her African

American cultural heritage. The fourth of Cross' stages is internalization, where racial identity conflicts are resolved and, in turn, the individual internalizes a positive African American identity. Finally, the fifth stage, internalization-commitment, involves a sustained interest in African American affairs and a long-term commitment to ethnic concerns. 
Over the next 20 years research on racial identity burgeoned within the social sciences. In response to the saturation of information, Locket and Harrell (2003) employed a hierarchical linear regression procedure to address the issue of over-interpretation in research on racial identity attitudes and academic achievement. They administered the Black Racial Identity Attitudes Scale (RIAS-B), the Rosenberg Self-Esteem Scale, and a background questionnaire to African American students attending an $\mathrm{HBCU}(n=128)$. Results showed that over $50 \%$ of racial identity's effect on academic outcome was predicted by individual differences in self-esteem. This research confirmed previous findings that the self-esteem of African Americans is inversely related to dominant pre-encounter attitudes and shares a positive association with immersion/emersion and internalization attitudes (Lockett \& Harrell, 2003; Phinney, 1996). In other words, the self-esteem of African Americans seems to emerge and strengthen as one progresses through Cross' (1991) stages.

At present, there is no available evidence that has linked internalization-commitment (i.e., the fifth stage) to self-esteem. Although the internalization attitudes (i.e., the fourth stage) have been described as a state governed primarily by the intellect (Cross, 1991), it is not certain what aspects of racial/ethnic identity translate into cognitive ability and academic performance. Therefore, a more complete description of ethnic identity appears valuable in promoting a greater understanding of the collegiate experience of African American students.

There are numerous models of racial and ethnic identity; however, the ethnic identity model created by Jean Phinney (1989) is among the most widely studied models of ethnic identity. According to Phinney (1996), the initial or first stage of ethnic identity, Unexamined Ethnic Identity, is characterized by a lack of exploration. In this stage, individuals may experience diffusion or foreclosure; that is, a lack of interest in ethnicity or a general acceptance 
of others' opinions. This stage of ethnic identity is described as a period when ethnicity is not yet perceived as significant and has thus received limited thought. The individual, typically a child or young adolescent, accepts the values and attitudes present in his or her environment. For example, when the African American family and community present a strong positive image of African Americans for a child, the child is likely to have a positive identification with the group, even though the group has not been consciously examined. On the other hand, children run the risk of internalizing negative stereotypes about African Americans from society.

The second stage of Phinney's 1996 model, Ethnic Identity Search, combines aspects of encounter and exploration, and the individual begins to truly develop his/her ethnic identity at this point. This stage can be seen as a period of search (Phinney, 1996) or immersion (Cross, 1991), wherein one becomes deeply interested in knowing more about African Americans. However, experience likely plays a more important role, as adolescents move into a larger world, encounter more people from diverse backgrounds (presumably different from their own), and are increasingly exposed to the likelihood of discriminatory experiences. Such experiences often trigger the desire to understand the history, traditions, and current situation of their group (Phinney, 1996). African American clubs on university campuses can assist students in this process, as can efforts by the educational institutions themselves to develop courses, invite speakers, and promote activities that give recognition to African Americans. Throughout this exploratory period, ethnicity is assumed to be highly salient and attitudes toward one's group generally appear highly positive.

The final stage of Phinney's (1996) model is Ethnic Identity Achievement. Individuals at this stage have a clear sense of their ethnic identity and are therefore more able to successfully navigate between cultures and identities. Ethnic identity achievement is displayed via the 
attainment of a secure, confident sense of oneself as a member of his or her group (Phinney, 1996). They feel secure in their own ethnicity and are assumed to hold a positive but realistic view of their own group. Individuals at this final developmental stage are assumed to have abandoned any potential anger toward the majority group and are generally open to other groups; however, their personal relationships with other groups may vary (Cross, 1991; Phinney, 1996).

Contemporary research on ethnic identity maintains that the previously described stages of ethnic and racial identity are important, both conceptually and experientially; however, they remain difficult to measure accurately, and there is a relative absence of sound empirical research to validate many of them (Cokley, 2007). Nevertheless, among the existing measurements of ethnic identity, the most commonly used is a questionnaire titled the Multigroup Ethnic Identity Measure (MEIM) (Phinney, 1992).

Rather than assessing stages, the MEIM is based on the conceptualization of ethnic identity as a continuous variable, ranging from a low or weak identity to a high, strong, positive identity. The questionnaire includes items that tap the extent of exploration and commitment regarding one's ethnicity and also the degree to which attitudes are positive. Additionally, this measure includes an assessment of attitudes toward, and interactions with, ethnic groups other than one's own (i.e., the Other Group Orientation (OGO) factor). The measure has been found to be reliable with a wide variety of ethnic groups, including African Americans, and can be incorporated as a tool for individual measurement across the life span (Phinney, 1992). Ethnic identity, as measured by this scale, appears to be a characteristic of an individual that can be reliably measured, that shows variation across both individuals and ethnic groups, and that is implicated in psychological wellbeing (Phinney, 1992; Phinney, 1996; Phinney et al, 2007). 
In 2006, Johnson and Arbona studied the extent to which ethnic identity and racial identity were related constructs among African American college students. Participants were 140 African American college students (about 70\% females) enrolled in two universities in a major city in the Southwest United States. Participants' ages ranged from 17 to 58 years

$(M=24, S D=6.8)$. In terms of educational level, $10 \%$ of the students were freshmen, $17 \%$ sophomores, $24 \%$ juniors, $19 \%$ seniors, $21 \%$ graduate students, and $9 \%$ did not indicate their year in school. Among the assessments administered were the Black Racial Identity Attitude Scale (RIAS-B) (Parham \& Helms, 1981), and the MEIM (Phinney, 1992). Results indicated that an endorsement of pre-encounter racial identity attitudes was negatively related to ethnic identity achievement, whereas endorsement of internalization racial identity attitudes was positively related to ethnic identity achievement. In other words, this finding of the Johnson Arbona (2006) study extends the support of the previously delineated relationship between ethnic identity achievement and racial identity attitudes for African American college students.

\section{Psychosocial Realities in Education}

Any discussion of the academic experiences of African Americans must begin with an acknowledgment of the work of John Uzo Ogbu. Ogbu was a prominent Nigerian-American anthropologist and professor; and he was best known for his theories on the observed phenomena involving race and intelligence $(1978 ; 1981)$. Ogbu was also a key innovator in the exploration of how racial and ethnic differences contributed to educational attainment and achievement.

According to Ogbu (1981), the social inequity experienced by Blacks in academia was partially attributable to a long-standing history of PWIs honoring various traditional ideas and behaviors, some of which involved prejudicial and discriminatory practices. Furthermore, Ogbu has suggested that as various types of outcome data became available as evidence of academic 
performance, a traditional view developed wherein one's overall ability and potential for success was inexorably linked to their academic achievements. Unfortunately, many of these performance measures, or data outcomes, conveyed a distorted perspective of the overall ability and potential of many Black students (Ogbu, 1981).

In most contemporary societies, performance indicators (e.g., GPAs, SAT scores, graduation rate, etc.) are used as a primary means to show discrepancies amongst both individuals and groups. Educational achievement and attainment in American society, in general, has well-established links to life outcomes such as enhanced life satisfaction and well-being (Chavous et al., 2003). Historically, many African American students encountered negative cultural stereotypes that portrayed members of their racial/ethnic group as less intelligent than their White counterparts (Mickelson, 1990; Ogbu, 1978; Ogbu, 1981; Steele, 1997). These stereotypes were compounded by statistics suggesting that, on average, members of ethnic minority groups score lower on achievement tests, have lower GPAs, and attain lower levels of education than their White peers (Fordham \& Ogbu, 1986; Mickelson, 1990; Steele, 1997). What is more, many intelligence tests (i.e., IQ tests) appear to be culturally biased (Brown, Reynolds, \& Whitaker, 1999; Sue, Ivey, \& Pedersen, 1996), which potentially distorts our understanding of the intelligence of African American students.

Although academic performance data and IQ scores may not always provide an accurate reflection of one's individual ability, they continue to be utilized as both a reliable and valid indicators of one's academic ability and potential for success (NCES, 2012). For many African American students, an additional challenge may exist wherein an individual and/or institution espouses the belief that his/her academic ability may be compromised by a lack of self-esteem or academic self-concept (Cokley, 2000; Fordham, \& Ogbu, 1986). Fortunately, Ogbu's work 
inspired many in the social sciences to explore perceived inequities and provided many additional insights into the overall experience of African Americans who receive an education in a predominantly White environment.

Gaps in school achievement and retention between White and African Americans, at all levels of schooling, have been strikingly consistent in American society for decades (Fordham \& Ogbu, 1986; Mickelson, 1990; Ogbu, 1981; Steele, 1992). However, recent national statistics suggest a reduction in this gap (2012, NCES). For example, over the past 20 years the national drop-out rate for African American college students (i.e., the percentage who did not complete college within a six-year window of time) decreased by $10 \%$; as it went from $70 \%$ in 1990 to 60\% in 2010 (American Council on Education, 1990; NCES, 2012). This is compared to a 2\% change in drop-out rate for White college students over the same 20 year period; as drop-out rates decreased only slightly from $42 \%$ in 1990 to $40 \%$ in 2010 (American Council on Education, 1990; NCES, 2012).

\section{Self-Esteem of African American Students}

Although one may be led to assume, through the perpetuation of negative stereotypes and the prevalence of relatively poor outcome data, that the individual self-esteem of African American students at PWIs would thereby be compromised, the opposite turns out to be true. Researchers have discovered that African American students have levels of self-esteem and academic self-concepts that are, on average, equal to or higher than those of White students (Cokley, 2000; Cokley et al., 2003; Graham, 1997; Phinney, Cantu, \& Kurtz, 1997; Schmader, Major, \& Gramzow, 2002).

Self-esteem, in particular, is a personality trait that has been extensively investigated in African Americans and White Americans. Self-esteem can be broadly defined as the positive or 
negative feelings one has about oneself (Richardson, Ratner, \& Zumbo, 2009). According to Moeller (1994), efforts to improve academic performance in children have often centered on raising their self-esteem with programs like "Project Follow Through," a federal program with a self-esteem component, to assist Head Start children in Grades 1 through 3. He concluded, however, that such efforts are misplaced because the research indicates that increased selfesteem rarely leads to improved academic performance.

In 1971 adolescent psychologist Allen H. Frerichs studied how self-esteem related to academic success for disadvantaged students. The subjects of his study were 78 African American sixth graders living in a large mid- western inner-city area. They attended a neighborhood elementary school and virtually all the children were from a low socioeconomic standing. The school- related factors tested were IQ test scores, GPA for the academic year, and standardized achievement reading score. Contrary to Moeller's (1994) conclusion, the Frerichs (1971) study supports the research that shows a relationship between a positive selfesteem and school success. Additionally, Hendrix-Wright (1981) found that African American youths who had both a high racial identification and an external locus of control had the highest self-esteem in their sample.

Phinney and Chavira (1992) conducted a longitudinal study of ethnic identity in African Americans, and determined that self-esteem and ethnic identity were significantly related to each other. A few years later, in a study of 9th- through 12th-grade African American adolescents, McCreary, Slavin, and Berry (1996) found that the racial identity variable of attitude toward other African Americans was statistically correlated with both self-esteem and problem behaviors. McCreary et al. suggested that a positive attitude toward other African Americans 
helped participants deal more effectively with stress and therefore was associated with higher self-esteem and fewer problem behaviors.

According to Baldwin (1984), the historical opinion that African Americans have low self-esteem was due to an erroneous utilization of a Euro-centric approach to conceptualize and explain the behavior of African Americans. In actuality, research in the social sciences continues to support the idea that African Americans have equal or greater self-esteem than Whites. For example, Tashakkori and Thompson (1991) found that African American adolescents have higher self-esteem than their White counterparts. Osborne (1995) also found that global selfesteem was higher for African American students than for White students in 8th- to 10th-grade students. Furthermore, Hughes and Demo (1989) argued that social contact with White Americans and the attitudes of White Americans are generally unimportant to African American self-esteem.

In 1992, African American psychologist Claude Steele observed a process occurring for African American students wherein they appeared to de-identify with academics as a way to maintain healthy self-esteem. "Academic disidentification is essentially a progressive state of being wherein the general self-concept becomes increasingly less associated with academic performance the longer one stays in school" (p. 70). According to Steele, all students start school identifying with academics; that is, they are motivated to do well, and their academic performance is related to how they feel about themselves. However, Steele observed a social psychology process occurring throughout the educational experience for African American students wherein the longer the individual remained in school the less likely they were to identify with academics as a salient part of their identity. Steele's work has also provided evidence that an individual's awareness of negative stereotypes is generally heightened as a result of their 
experiences with adverse individual encounters (e.g., teacher bias and lack of culturally relevant material).

In a later study, Steele and Aronson (1995) identified a similarly occurring psychosocial process they labeled "stereotype threat" (p. 614). Stereotype threat is suggested to function in such a way that African American students begin to fear their behavior (i.e., lower academic performance) will confirm the stereotypes associated with their group. Therefore, to protect one's own self-esteem from being diminished, an African American student may deidentify with academics (Steele, 1997). Their self-esteem, while still high, is no longer contingent upon and related to their academic performance.

Steele and Aronson (1995) found that, with SAT differences statistically controlled, African American participants performed worse than White American participants when the test was presented as a measure of their ability but improved dramatically, matching the performance of Whites, when the test was presented as less reflective of ability. Thus, stereotype threat can impair the intellectual test performance of African American students, and lifting the threat has the potential to improve that performance.

It is assumed that one way in which African Americans might cope with academic threats is by limiting the sense of self worth they derive from their academic outcomes. Steele (1997) used the term "psychological disengagement" to describe this coping tool: Psychological disengagement is a self-defense strategy used to protect one's self-esteem from academic performance so that self-esteem is not contingent upon one's successes or failures in academia. As part of a larger class of self-protective strategies, psychological disengagement is more likely to occur in evaluative situations (e.g., a classroom or testing environment) that might threaten a person's view of self (Schmader, Major, \& Gramzow, 2001). 
In 2007, Cokley and Moore conducted a study exploring the moderating and mediating effects of gender and psychological disengagement on the academic achievement of African American college students. They discovered that for African American female students, an achieved ethnic identity was positively correlated with academic self-concept; whereas among African American male students, no relationship between ethnic identity and academic selfconcept was found (Cokley \& Moore, 2007). In other words, the experience of psychological disengagement has varying effects on the ethnic identity and academic self-concept of African American college students, and some of this difference is purportedly influenced by gender.

Psychologists should therefore be familiar with gender differences in stereotype threat response and be comfortable to explore with African American clients how it may operate to suppress their academic performance (Cokley \& Moore, 2007; Steele \& Aronson, 1995). This is a critical point because a psychologist unaware of stereotype threat could mistakenly conceptualize an African American student's passive attitude about his/her potentially mediocre academic performance as evidence of a lack of intrinsic motivation (Cokley, 2002). This conceptualization would most likely lead the psychologist to try to facilitate the student to be more intrinsically motivated while potentially being unaware of the student's possible feelings of self-doubt that may be driven by an unspoken fear of confirming negative stereotypes of African American intellectual inferiority (Steele, 1992).

A similar psychosocial phenomenon was observed in a study by Graham (1997), when she explored the motivational factors influencing group identification amongst African American adolescents. Graham discovered that African American adolescent females admire, respect, and want to be like other African American females, who try hard, and do well in school. However, unlike the females, the African American adolescent males had a reduced likelihood of selecting 
high-achieving African American male classmates as the ones they admire, respect, and want to be like. Among the males, this display of relative indifference is believed to be reflective of the achievement behaviors that are valued by the society; and, may be one self-esteem protecting mechanism by which they cope with the dual stresses of academic challenge and experienced prejudice.

In addition to the previously identified self-esteem protecting mechanisms, a construct Chavous (2000) labeled higher "racial centrality" (p. 99), has been observed to be positively related to the ethnic fit of African American college students on predominantly White campuses. Racial centrality is defined as the extent to which one maintains group pride (i.e., private regard) and positive beliefs about society's views of African Americans (i.e., public regard). Chavous et al. (2003) discovered higher racial centrality was related to more positive academic beliefs, supporting the motivational perspective on the role of group identification (Graham, 1997).

Furthermore, the findings of Chavous et al. (2003) suggest that African American youth who possess positive societal views about African Americans show stronger school attachment. On the other hand, African American youth with lower group affiliation, lesser group pride, and more negative societal perceptions showed the highest number of students out of school in 12 th grade, and the lowest college attainment. These findings are not consistent with the disidentification perspective; instead, they reflect the more protective nature of the motivational perspective on group identification (Graham, 1997), where connecting with one's own ethnic and racial group is related to discrimination awareness in ways that may facilitate academic success.

Although there is some debate as to what psychological processes promote/inhibit wellbeing and academic achievement for African American students, the study of achievement values and how they get expressed in the broader context of social influences (e.g., peers, 
teachers, and parents) remains an untapped source of information with regards to the motivational processes of African American students (Graham, 1997). In order to better understand the dynamic processes that contribute to individual espousal and enactment of academic values, we must develop an understanding of academic selfconcept and how it functions for African American college students.

\section{Role of Academic Self-Concept}

Academic self-concept can be broadly defined as a student's views about his/her academic ability as compared to other students (Cokley, 2000). Specifically, academic selfconcept is defined as "attitudes, feelings and perceptions relative to one's intellectual or academic skills" (Lent, Brown, \& Gore, 1997, p. 308). Multiple studies have demonstrated that academic self-concept is positively related to academic achievement among African American college students (Awad, 2007; Cokley, 2000, 2002; Gerardi, 1990; Reynolds, Ramirez, Magrina, \& Allen, 1980).

Gerardi (1990) stated that African American students at HBCUs tended to have generally higher academic self-concepts when compared to African American students attending PWIs. Gerardi also concluded that although cognitive measures, such as the SAT, have traditionally been better predictors of GPA than measures of academic self-concept, his results support the converse (i.e., that academic self-concept is a better predictor of GPA). What is more, using GPA as a measure of academic achievement, across institutions, may pose another limitation because of the different levels of academic ability amongst incoming students and the different institutional norms regarding academic competition (Cokley, 2000).

In 1992, as part of a symposium targeting minority participation in higher education, a highly published African American sociologist named Walter R. Allen stated, 
To date, research on Black students in U.S. higher education has generated as many questions as answers. There is a particular need to understand the effects of individual and institutional characteristics on student outcomes and, at the same time, to explore the relative importance of more proximate factors versus more distant factors as explanations for differential achievement by Black college students. (p. 32)

Allen (1992) studied the college experiences of 953 African American undergraduate students who attended HBCUs and 1,578 who attended PWIs. The effects of predictor variables were investigated by borrowing from related studies and analyzing data from the 1991 National Study on Black College Students. Allen found that African American students attending PWIs reported less favorable relations with professors when compared to their counterparts attending HBCUs. Academic achievement was highest for subjects who had high academic self-concept, were certain that their college choice was correct, and reported positive relationships with faculty. African American students attending PWIs reported lower academic achievement and self-concepts, as well as lower levels of social involvement. Results suggest that outcomes were influenced by the immediate social context, while interpersonal relationships bridged individual disposition and institutional context.

Basically, the institutional setting, social environment, and quality of the student-faculty relationship appear to have a cumulative impact on the academic self-concept of African American college students. Although there is research suggesting that HBCUs seemingly offer an environment uniquely conducive to the promotion of academic achievement for African American students, it should also be noted that Allen (1992) reported campus racial composition to be only the second strongest predictor of academic achievement while good relations with faculty members emerged as the strongest predictor. 
Nevertheless, contrary to what Allen's (1992) study suggests, Cokley (2000) examined 206 African American students attending HBCUs and PWIs and found there was no significant difference in the academic self-concept of African American students attending PWIs versus African American students attending HBCUs. However, there were institutional differences in cumulative GPA, quality of student-faculty relationships, and perceptions about fairness of evaluation of an African American student's academic performance. Students attending HBCUs reported higher GPAs, higher quality of student-faculty relationships, and more positive perceptions of the evaluations of their academic performance. Overall, students attending HBCUs reported more positive experiences and perceptions of their environment than their peers attending PWIs.

Additional research measuring the academic self-concept of African American college students at PWIs has revealed that African American students' self-doubt appears to involve their beliefs about academic preparation, not natural abilities (Cokley et al., 2003). In the Cokley et al., (2003) study, they found that self-doubt regarding one's natural abilities was unrelated to discouragement about school. One interpretation of this finding is that when an African American college student gets discouraged about school, the discouragement is not because he/she doubts his/her abilities, but because he/she is disappointed in the actual outcome. In addition, research on college students has demonstrated that African American college students, when compared to White college students, tended to develop negative performance expectations about school while simultaneously being satisfied with their efforts (Cokley, 2002). This implies that negative expectations regarding academic performance are related to, but separate from, one's belief about his or her efforts. 
Witherspoon, Speight, and Thomas (1997) reported that an internalized racial identity (i.e., Cross' fourth stage) presents a positive relationship with academic self-concept. From this perspective, internalization attitudes promote self-worth that may encourage students to try things simply because they seem interesting, without fear of failing. As a result, students may feel capable of analyzing and solving novel problems and may approach educational environments with a more positive outlook. A positive outlook on education appears to be crucial for African American students' academic performance, because negative experiences in school are assumed to influence an increase in one's tendency to form a reactionary personal identity (Steele \& Aronson, 1995).

Witherspoon et al. (1997) examined the extent to which racial identity, self-esteem, and academic self-concept were related to academic achievement for 86 African American high school students in Upward Bound programs at two medium-sized universities in the Midwest. Project Upward Bound was a national pre-collegiate program funded by the U.S. Department of Education that's curriculum was designed to encourage the development of the motivation and skills needed for success in school. Although all Upward Bound students from the two programs were invited to participate in the study, only respondents who identified as Black or African American were used in this study. Of the individuals studied, $35 \%$ of the participants were male $(n=30)$ and $65 \%(n=56)$ were female. The students' ages ranged from 12 to 18 years-old, with the average age of 15.4 years. Parental consent was therefore attained for all of the research participants. No financial or credit incentives were given for participation; and, the project administrator of each respective Upward Bound program supplied the GPAs of participants, which were then used as a measure of school performance. 
Upward Bound students from the first university were introduced to the research study at a parent's orientation day. Parents were able to turn in consent forms to the investigators that day. At one of their subsequent weekly meetings, students were administered the self-report surveys comprised of the 30-item RIAS-B (Parham \& Helms, 1981), the 16-item Personal Orientation Inventory (POI)/Self-Regard subscale (POI; Shostrum, 1963), and the 40-item Academic Self-Concept scale (ASCS; Reynolds et al., 1980) as a group. Students were given up to 45 minutes to answer all the items. Follow-up phone calls were made by the investigators, as needed, only to gather missing parent or guardian consent forms (Witherspoon et al., 1997).

The Upward Bound students at the second university were investigated using an alternate procedure than the first participant pool. In this sample, the students were given individual packets by program administrators that contained the aforementioned surveys, as well as parent or guardian consent forms. The only directions given were to complete the surveys as thoroughly as possible and to return the completed packet, along with the consent form, to the Upward Bound office. It is possible that this procedural inconsistency in data collection could have altered the results of the Witherspoon et al. (1997) study. What is more, the investigators' utilization of the 16-item Self-regard subscale of the POI as a pure measure of self-esteem is questionable (Shostrum, 1963).

Nevertheless, a multiple regression analysis was conducted by the investigators which indicated GPA was best predicted by immersion racial identity attitudes and academic selfconcept. Interestingly, none of the various racial identity attitudes were significantly correlated with academic self-concept or self-esteem (Witherspoon et al., 1997). This is surprising given the existent empirical support, which generally demonstrates a positive relationship between racial/ethnic identity and self-esteem, as well as with academic self-concept. Therefore, the 
proposed study is a replication of the Witherspoon et al. (1997) study of African American high school students using alternate measures of racial/ethnic identity and self-esteem (i.e., the MEIM and RSES as opposed to the RIAS and POI), as well as an assessment of the generalizability of the Witherspoon et al. (1997) study by investigating a college student population.

\section{Summary and Conclusion}

For African American students at PWIs, the degree of experience with regards to interpersonal and social engagement has been shown to vary widely. It is possible that issues of cultural misrepresentation and the resultant power differentials existent in a PWI setting may compromise some individuals' ability to engage effectively within the environment.

Furthermore, the realities associated with being in a predominantly White environment can pose some limitations on the control African American college students may perceivably have over their experience. However, it appears that for African American students, ethnic identity can have a generally mediating effect on self-esteem and academic self-concept.

Based on the fact that African American students are often a considerable numerical minority at PWIs, they are more likely to engage in intergroup exchanges which, in turn, may increase their vulnerability to the problems associated with interracial communication and prejudice (Cole \& Yip, 2008; Dovidio, Gaertner, Kawakami, \& Hodson, 2002; Sue et al., 2007). Furthermore, the potential for avoidance of intergroup interaction may be a realistic strategy for members of the majority group at PWIs; but it is a much less viable option for groups constituting only a small proportion of the population (i.e., African Americans) (Cole \& Yip, 2008; Sue et al., 2007).

What is more, as college is often the time in which individuals openly explore their career options and lifestyle choices, it appears that ethnic identity would be particularly salient 
throughout ones collegiate experience. Likewise, an achieved ethnic identity could provide a valuable advantage to African American students attending PWIs. Existent research indicates that African American students do not have lower self-esteem than White students (Awad, 2007; Lockett \& Harrell, 2003; Negy et al., 2003), and the self-esteem of African American students is also unrelated to their grades (Cokley, 2000; Cokley et al., 2003). Thus, psychologists dealing with academic problems of African American students should likely place less emphasis on self-esteem when, potentially, more productive efforts (e.g., focusing on the fear of confirming negative stereotypes, and/or targeting the elimination of campus racism and discrimination) could be pursued. It has also been recommended that psychologists focused on helping African American college students, particularly at PWIs, develop and nurture positive relationships with faculty, as this has been shown to be a valuable resource for the African American students who established such relationships (Cokley, 2000).

In 2003, Ogbu conducted research in the predominantly Black Cleveland suburb of Shaker Heights, Ohio. His research effort provided the community of Shaker Heights with educational policy recommendations that included the following: 1) more academic counseling, 2) a focus on teaching youth good study habits, and 3) identifying successful Black role models. Ogbu believed that anyone who professed to care about so-called "minority education" advocated these practices (Foley, 2005, p. 651). Ogbu also advocated for a series of teacher workshops focused on teacher expectations for African American students, and to create greater trust and community/parental involvement in schooling.

Predominantly White Institutions provide for a distinctively challenging social reality; and, there is ostensibly value in Phinney's (1996) model of ethnic identity, particularly as it relates to the engagement and success of African American students at PWIs. Since the 
emergence of multiculturalism in the counseling psychology literature (i.e., the past 20 to 30 years), much attention has been focused on the psychological importance of developing and maintaining a positive group identity (Cokley, 2005; Cole \& Yip, 2008). As a result, ethnic identity has emerged as an important multicultural and psychological construct. For African American college students, the development of ethnic identity is clearly influenced by an array of experiences occurring at the family, community, and societal levels, but specific factors that bring about transitions have yet to be identified (Cokley et al, 2003; Phinney, 1996).

Due to the individually motivated nature of ethnic identity development, student affairs professionals at PWIs (i.e., teachers, administrators, psychologists, counselors, etc.) may help African American students most by incorporating strategies intended to promote self-reflection and awareness. Although it may require the collective effort of students, parents, faculty, and other support staff, PWIs can potentially do more to promote the optimum functioning of their African Americans students.

What is more, the promotion of a cooperative effort within PWIs will likely require a valuing of collectivism. The idea of collectivism primarily relates to the concept of interdependence, where the psychological unit of identity resides within the family, group, or society (Sue, Ivey, \& Pedersen, 1996). This worldview is more common in Eastern cultures (e.g., Asian culture), and is also prevalent among African Americans in the United States (Cokley, 2005). For that reason, PWIs may benefit from promoting sensitivity to the collectivistic worldviews of their students; otherwise, African American students may risk being perceived by the traditional Western individualistic standards as dependent, immature, and unhealthily enmeshed. 
In sum, there are various social, political, cultural, and other realities which influence the African American college student experience at PWIs. While some of the experiences accompanying PWI environments may potentially contribute negatively to the development of African American students, PWIs also provide an increased opportunity for adaptive crosscultural encounters and developmental experiences that could have a significantly positive effect on personal growth.

Numerous psychologists and social scientists alike have determined there is a particular need to understand the impact of individual characteristics and institutional differences on African American student outcomes (Allen, 1992; Cokley, 2002; 2005; Cole \& Yip, 2008; Lockett \& Harrell, 2003; Phinney et al., 2007; Thompson, Andersen, \& Bakeman, 2000). Collectively, cultural researchers have broadly challenged those in the social sciences to study the relative importance of both proximate and more distant factors as explanations for differential achievement by African American college students. This dissertation is, in part, a response to this challenge; alternatively, this proposed study is intended to replicate and expand the findings of Witherspoon et al. (1997).

Considering the fact that African American college students attend PWIs at a rate higher than any other numerical minority (NCES, 2014), it is advisable that helping professionals and educators alike do their part to better understand the impact a predominantly White environment potentially has on these students. Thus, the general purpose of this study is to explore the psychosocial impact of several factors, both dispositional (e.g., self-esteem, academic selfconcept, and ethnic identity) and situational (e.g., racial composition of the institution), thought to influence the experiential reality for African American college students. 
In this dissertation the variables of ethnic identity, self-esteem, and academic selfconcept will be studied in relation to African American student's experiences at a PWI. The following questions will be answered: Are differences in self-esteem and academic self-concept significantly related to variations in ethnic identity among African American students attending a PWI? If so, what is the relationship between ethnic identity, self-esteem, academic selfconcept, and grade point average (GPA); and, what is the best predictor model for GPA?

The resultant hypotheses will be tested:

1. African American students at a PWI with higher MEIM factor scores (i.e., EI \& OGO scores) will report higher RSES scores than African American students with lower MEIM factor scores.

2. African American students at a PWI with higher MEIM factor scores will report higher ASCS scores than African American students with lower MEIM factor scores.

3. African American students at a PWI with higher MEIM factor scores and higher ASCS scores will report higher GPA's than African American students with lower MEIM factor scores and lower ASCS scores. 


\section{CHAPTER III}

\section{METHOD}

The purpose of this study was to contribute to a deeper understanding of the various factors shown to influence the African American student experience at predominantly White institutions (PWIs). African American college students attend PWIs at a rate higher than any other numerical minority (NCES, 2014); and, despite their increased enrollment at PWIs, HBCUs have shown greater improvements in African American student graduation rates (Toldson \& Cooper, 2014). As a result, professionals and educators alike have been challenged to develop a better understanding of the influences a predominantly White environment potentially has on the academic and interpersonal experiences of African American college students. In this study, the variables of ethnic identity, self-esteem, and academic self-concept were examined among a sample of African American college students attending a PWI. A modified replication of the Witherspoon et al. (1997) study of African American high school students was conducted, using alternate measures of racial/ethnic identity and self-esteem; and, the generalizability of the Witherspoon et al. (1997) study was challenged by investigating a college student population. Thus, the following research questions were examined:

- Are the self-esteem and academic self-concept of African American college students at a PWI related to individual variations in ethnic identity?

- What is the relationship between ethnic identity, self-esteem, academic self-concept, and grade point average (GPA); and, what is the best predictor model for GPA?

\section{Design}

To answer the first question above, a correlational study was conducted incorporating cross-sectional survey research with African American college students attending a PWI. A 
series of one-way ANOVAs were conducted to assess for possible sex, age, and class year differences on all predictor and criterion variables. The second question, which predicts the impact of variables, was analyzed through the use of a forced-entry multiple regression analysis. Volunteer survey questionnaires were administered and collected via internet. An integration of both partial and multiple correlations determined the relationship(s) between variables.

The correlational design allowed for an examination of the relationship between academic self-concept, self-esteem, and ethnic identity; in addition, a regression analysis was conducted to determine the viability of a predictor model for GPA based on the potentially causative relationships between the variables. Therefore, both an explanatory design (i.e., the extent to which academic self-concept, self-esteem, and ethnic identity are related) and a prediction design (i.e., the degree to which each variable positively predicts GPA) were employed.

Lastly, a brief measure of social desirability (i.e., the Marlowe-Crowne Social Desirability Scale: Short Form C (MC-C)) was introduced, to explore the response tendencies of participants. Individuals who present in a socially desirable manner attempt to appear overly moral, honorable, and virtuous by denying common yet undesirable traits (e.g., jealousy or anger), and/or exaggerating uncommon yet desirable traits (e.g., never being late or never lying to others) (Crowne \& Marlowe, 1960). Thus, all participants were administered a demographic data questionnaire (to determine age, sex, race/ethnicity, class year, major and current GPA), the MEIM, the RSES, the ASCS, and the MC-C. The order of administration remained consistent.

\section{Participants}

African American on-campus students enrolled at a mid-sized, predominantly White institution in the Mid-Atlantic region of the United States were studied. According to the NCES 
(2012), approximately $86 \%$ of the students enrolled at the studied university during 2011 (or 20,609 out of 23,918) identified as White. Participants were recruited via the university's electronic messaging system, facilitated by the office of the university registrar. This has been done by other researchers in university settings (Cokley, 2000; Cole \& Yip, 2008).

Sample size and power. Witherspoon et al. (1997) utilized a medium effect size in conducting a power analysis for their research. Therefore, a medium effect size was used in determining a sample size for the current study. According to Cohen (1992), a sample size of 76 participants is required given an alpha level of .05 and beta level of .2 .

\section{Variables}

Ethnic identity functioned as an independent and explanatory variable in this study, whereas self-esteem and academic self-concept were approached as both dependent and independent variables. In other words, academic self-concept, ethnic identity, and self-esteem all served as independent variables insofar as they were each found to predict GPA. Multiple oneway ANOVAs were conducted to assess for possible sex, age, and class year differences on all predictor and criterion variables. As an explanatory variable, the ethnic identity (i.e., MEIM scores) of African American students was compared to self-esteem (i.e., RSES scores) and academic self-concept (i.e., ASCS scores) by way of the Pearson product-moment correlation coefficient, or Pearson's $r$ to measure the degree of linear dependence between variables.

Social desirability functioned as a moderator variable; and, can be defined as the tendency to give overly positive self-descriptions (Paulhus, 2002). The need to examine social desirability as a response tendency with self-report measures (e.g., the MEIM, RSES, \& ASCS) has been well documented and continues to be a methodological consideration in both research and practice (Greenberg \& Weiss, 2012; Paulhus, 2002; Reynolds, 1982). 
Furthermore, because an additional purpose of the study was to test a model that would predict GPA (i.e., the criterion variable), a simultaneous multiple regression analysis for GPA was calculated with ASCS scores, RSES scores, and the MEIM factor scores as predictor variables. A forced-entry method was incorporated over a step-wise method to examine the combined influence of the variables of interest.

\section{Measures}

Multigroup Ethnic Identity Measure (MEIM; Phinney, 1992).

The Multigroup Ethnic Identity Measure focuses on ethnic identity as a general phenomenon that is relevant across a wide range of groups. Self-identification and ethnicity, ethnic behaviors and practices, affirmation and belonging, and ethnic identity achievement were chosen as common components of ethnic identity. Additionally, this measure includes an assessment of attitudes toward, and interactions with, ethnic groups other than one's own. Following the model of the Objective Measure of Ego Identity Status (Adams et al., 1987), an initial version of the scale was developed to assess ethnic identity search and commitment and was administered to 60 college undergraduates as a trial measure (Phinney \& Ambarsoom, 1987).

To broaden the measure to include the major components of ethnic identity that have been assessed in other research, existing literature was reviewed (Phinney, 1990). At the same time, a series of interview studies was conducted to examine adolescents' spontaneous comments indicative of different aspects of ethnic identity (Phinney, 1989; Phinney \& Tarver, 1988). After initial revision, items were added to assess ethnic attitudes (belonging, affirmation, and denial) and ethnic behaviors. Items were revised on the basis of item analysis to arrive at the 20 -item questionnaire. 
An exploratory factor analysis was conducted using squared multiple correlations as estimates of communalities. For a multi-ethnic high school sample, using the proportion criterion, three factors were indicated. However, two of the factors were subfactors of the first factor and were highly correlated. For a college sample, using the proportion criterion, five factors were indicated. But, three of these were highly inter-correlated, and the remaining two appeared to be subfactors of the Other-Group Orientation (OGO) Scale. A two-factor solution was therefore chosen.

Reliability coefficients (Cronbach's alpha) were calculated separately for the measure of ethnic identity and two of its subscales, as well as for the measure of other-group attitudes. Overall reliability of the 14-item Ethnic Identity Scale was .81 for a high school sample and .90 for a college sample. For the 5-item Affirmation/Belonging subscale, reliabilities were .75 and .86 for the high school and college samples, respectively. For the 7-item Ethnic Identity Achievement subscale, reliabilities were .69 and .80 , respectively. The 6-item Other-Group Orientation (OGO) scale displayed a reliability of .71 for high school students and .74 for college students. No coefficients were given for the Ethnic Behaviors subscale, because reliability cannot be calculated with only two items. However, separate analyses showed that the ethnic behavior items increased the overall reliability of the measure.

Rosenberg Self-Esteem Scale (RSES; Rosenberg, 1965).

Originally intended as a measure of self-esteem for adolescents, Rosenberg's Self-Esteem Scale is probably the most widely used measure of self-esteem for adult populations. The scale is composed of 10 items, five of which are negatively worded. Although originally constructed as a Guttman-type scale (i.e., items with an ordinal pattern on the attribute), most researchers use a four-point response format ranging from strongly agree to strongly disagree. Rosenberg's Self- 
Esteem Scale is the standard measure of self-esteem in psychological research. The scale provides a short, straightforward, and convenient method for measuring global selfesteem. Because self-esteem is a trait-like construct, this scale may be of interest to researchers as a moderator variable in research rather than a dependent variable.

Numerous studies have supported the reliability of the RSES across a variety of populations. Rosenberg (1979) reported an internal consistency of the RSES with a coefficient of reproducibility of $92 \%$. Silber \& Tippet (1965) reported test-retest reliability of .88 over a twoweek period for a sample of college students. Fleming and Courtney (1984) reported a 1-week test-retest reliability coefficient of .82 , and an internal consistency, or coefficient alpha, of .88 . As far as convergent validity, Reynolds (1988) found a .44 correlation between the RSES and the Academic Self-Concept Scale (Reynolds, 1988). A discriminant validity check revealed no relationship between GPA and scores on the RSES. Scores on the RSES were also unrelated to locus of control, SAT scores, and social desirability. This is expected because the scale is designed to measure general self-concept and not specific facets (Reynolds, 1988).

Conceptually, the RSES was constructed as a one-dimensional scale, measuring selfesteem as a global construct. Rosenberg (1965) viewed self-esteem as a one-dimensional idea that reflects either a positive or negative orientation towards self. A cumulative score is derived by reversing the five negatively worded items and adding them with the five positively worded items. Psychometric studies have largely supported the unidimensionality of the RSES (Corwyn, 2000; Dunbar, Ford, Hunt, \& Der, 2000).

Corwyn (2000) used confirmatory factor analysis to examine the factor structure of the RSES in several samples of English speaking participants. The RSES represented a onedimensional construct of global self-esteem, but was often confounded by method effects 
associated with the negatively worded items. Schmitt \& Allik (2005) translated the RSES into 28 languages and administered it to 16, 988 respondents across 53 different countries. Principal components analysis was used to investigate the factor structure. In most countries, a onedimensional structure was produced due to all items loading highly on the first principle component.

Academic Self-Concept Scale (ASCS; Reynolds, Ramirez, Magrina, \& Allen, 1980). The Academic Self-Concept Scale is a 40-item scale that measures the academic aspect of the general self-concept among college students. The instrument uses a Likert-type scale ranging from 1 (strongly disagree) to 4 (strongly agree), and the global scores from this scale indicate academic self-concept. Scores can range from 40 to 160; the higher the score, the stronger the level of academic self-concept. The ASCS has been found to correlate with GPA $(r=.40-.52)$, and the Rosenberg Self Esteem Scale ( $r=.45)$ (Reynolds e al., 1980). A multiple regression analysis of the ASCS with GPA and Rosenberg scores as predictor variables resulted in a multiple correlation of .64.

Factor analysis of the ASCS yielded a seven-factor solution that accounted for $52.6 \%$ of total variance (Reynolds, 1988). The ASCS yields one global score and seven subscale scores. The items are keyed in a positive direction for academic self-concept. Reynolds tentatively named the seven factors the following: Grade and Effort Dimension (8 items), Study Habits/Organizational Self-Perceptions (6 items), Peer Evaluation of Academic Ability (8 items), Self-Confidence in Academics (3 items), Satisfaction with School (4 items), Self-Doubt Regarding Ability (8 items), and Self-Evaluation with External Standards Dimension (3 items). The scale yields a global academic self-concept score and seven subscale scores; however, 
perhaps because of the tentative description of the factors, researchers have only used the global score (Cokley, 2000; Lent et al., 1997).

Reynolds et al. (1980) reported a 2-week test-retest reliability coefficient of .88, and an internal consistency of .91 for the ASCS. The initial item pool consisted of 59 items. An itemtotal score correlation of .30 was selected as a minimum level for item inclusion. The reliability coefficients for the subscales range from .59 to .92 (Reynolds et al., 1980). Validation of the scale was achieved through the administration of the instrument to undergraduate college students in New York $(n=427)$ who were predominantly White; convergent validity, after correction for attenuation, was reported to be .44 (Reynolds et al., 1980). However, the scale has been administered to ethnic minorities including African Americans and students from various geographical regions and states in the U.S. since its development (Cokley, 2000; Zorich \& Reynolds, 1988). Sample items include: (a) if I try hard enough, I will be able to get good grades; (b) I often expect to do poorly on exams; (c) all in all, I feel I am a capable student; (d) at times I feel like a failure; and (e) I enjoy doing my schoolwork.

Cokley et al. (2003) designed a study to compare two ethnically homogenous college student samples, White American $(n=291)$ and African American $(n=396)$, to determine if a similar factor structure (regarding ASC) existed in each sample. This study extended the psychometric investigation of the ASCS conducted by Reynolds (1988). For the White American sample, six of the factors were similar to what Reynolds (1988) identified, while one was different. The African American sample yielded an eight factor structure, with five factors similar to what Reynolds (1988) identified, and 3 being different.

Cokley et al. (2003) labeled the eight factors of academic self-concept for African Americans the following: Self-Doubt Regarding Ability (6 items), Study Habits (5 items), 
Evaluation of Academic Ability (3 items; formerly labeled by Reynolds (1988) as the Peer Evaluation of Academic Ability factor), Self-Confidence in Academics (4 items), Negative Performance Expectation (2 items), Discouragement About School (3 items), Rewarded Efforts (3 items), and Satisfaction with Grades (2 items). These eight subscales have slightly lower alpha values ranging from .57 to .76 , as well as fewer items per subscale, with total items ranging from two to six. Rewarded Efforts (RE) had the highest mean value (3.47) and Study Habits (SH) had the lowest mean value (2.10). Two subscales were exclusive to the African American sample, Negative Performance Expectation (NPE; $M=3.3$ ) and Discouragement About School (DAS;

$M=3.18$ ). Higher scores on NPE equates to a pessimistic belief in school performance; where higher scores on the DAS factor equates to greater discouragement about school overall.

By using two ethnically homogenous samples, some important findings about academic self-concept emerged. First, factor analysis of ASCS with ethnically homogenous samples may result in different factorial structures. Second, and more importantly, differences in factor structure have implications for theoretical understandings of a psychological construct. In this study, the results indicated that academic self-concept is more differentiated among African American students than White American students. By showing that ethnically homogenous samples yielded different factor structures, it was suggested that using an ethnically heterogeneous sample may not always be methodologically sound, particularly when it masks important differences in understanding an important psychological construct such as academic self-concept. Therefore, in evaluating the data for the proposed study, the eight factor structure of the ASCS (i.e., the factor structure unique to African American populations) was applied to compare the academic selfconcept of students attending either a PWI or an HBCU. 
Marlowe-Crowne Social Desirability Scale (MCSDS): Short Form C (MC-C; Crowne \& Marlowe, 1960; Reynolds, 1982).

Of the instruments available to assess social desirability, the Marlowe-Crowne Social Desirability Scale (MCSDS; Crowne \& Marlowe, 1960) has been the most frequently used in clinical and research settings (Ballard, 1992; Beretvas, Meyers, \& Leite, 2002; Greenberg \& Weiss, 2012; Reynolds, 1982). The Marlowe-Crowne Social Desirability Scale has undergone extensive research, being listed in over 1,000 articles and dissertations (Beretvas et al., 2002). It is a self-report instrument that can be easily and quickly administered, and exhibits acceptable reliability and validity in a number of different samples (Davis \& Cowles, 1989; Loo \& Thorpe, 2000).

The Marlowe-Crowne Social Desirability Scale is also a public domain instrument, making it cost effective for users. A number of factor analyses have been conducted on the MCSDS, producing a variety of shortened versions (Reynolds, 1982; Strahan \& Gerbasi, 1972). The original version of the MCSDS is comprised of 33 items, whereas the scale of interest for this study is the shortened 13-item version (i.e., the MC-C) proposed by Reynolds in 1982.

The Reynolds short form C of the MCSDS provides a brief and simple measurement of social desirability (Reynolds, 1982). On the basis of reliability and validity results, Reynolds (1982) discovered that amongst the three studied short form options, the 13-item MC-C was the most psychometrically sound. The reliability coefficient (Cronbach's alpha) for the MC-C was

.76, which compares favorably to the original MCSDS (Crowne \& Marlowe, 1960; Reynolds, 1982). Concurrent validity was examined by Reynolds (1982) via correlations between the Marlowe-Crowne short forms (i.e., A, B, \& C) and the original MCSDS. The short form $\mathrm{C}$ correlated most highly with a .86 coefficient of determination. 


\section{Procedure}

African American undergraduate and graduate/professional students were introduced to the research by way of an e-mail from the author, sent via the university's electronic messaging system (see Appendix F). Participants were provided surveys electronically (through Qualtrics), and were given up to 30 minutes to answer all items. Upon completion of the survey, participants were given the option to enter a raffle for 1 of $10, \$ 20.00$ gift cards, to be delivered to a personal e-mail of their choice. The only directions given were to complete surveys as thoroughly as possible, and to provide a personal e-mail if interested in the raffle.

\section{Hypotheses}

Hypothesis I: African American students at a PWI with higher MEIM factor scores will report higher RSES scores than African American students with lower MEIM factor scores.

Hypothesis II: African American students at a PWI with higher MEIM factor scores will report higher ASCS scores than African American students with lower MEIM factor scores.

Hypothesis III: African American students at a PWI with higher MEIM factor scores and higher ASCS scores will report higher GPA's than African American students with lower MEIM factor scores and lower ASCS scores. 


\section{CHAPTER IV}

\section{RESULTS}

In this chapter the results of the data analyses are presented. The data were collected and then processed in response to the problems posed in chapter 1 of this dissertation. African American college students attend PWIs at a rate higher than any other numerical minority (NCES, 2014); and, despite their increased enrollment at PWIs, HBCUs are showing greater improvements in African American student graduation rates (NCES, 2014). As a result, professionals and educators alike have been challenged to develop a better understanding of the influences a predominantly White environment potentially has on the academic and interpersonal experiences of African American college students. Thus, the general purpose of this study was to explore the psychosocial impact of several factors, both dispositional (e.g., self-esteem, academic self-concept, and ethnic identity) and contextual (e.g., racial composition of the institution), thought to influence the experiential reality for African American college students. These objectives were accomplished. The findings presented in this chapter demonstrate the potential for merging theory and practice.

\section{Response Rate}

Nine-hundred and ninety-three survey invites were electronically sent to African American undergraduate and graduate/professional students enrolled at a PWI in the midAtlantic region of the United States (see Appendix F). Eligible participants were all determined to be on campus students, at least 18 years of age or older, and self-identified as African American (either singularly or in part) upon enrollment, as confirmed by the Office of the University Registrar. Of the 993 e-mailed surveys, 128 (13\%) were initiated and 91 (9\%) were completed. Of the 128 initiated surveys, 38 participants only partially responded to the initial 
measure (i.e., the ASCS) prior to aborting the survey entirely; therefore, greater than $10 \%$ of the responses were left blank rendering these surveys invalid in the ensuing analyses. Thus, 91 surveys were considered to be legitimate for this research and subsequently used in the analyses.

\section{Demographics}

Of the 91 participants, 59 (65\%) identified as female, $23(25 \%)$ male, and $9(10 \%)$ did not report sex. In terms of class year, 19 (21\%) identified as freshmen, 9 (10\%) sophomores, 15 (16\%) juniors, $18(20 \%)$ seniors, and 30 (33\%) identified as graduate/professional students. There was considerable diversity amongst the identified current or intended majors, with 53 uniquely endorsed undergraduate and graduate majors (see Appendix G). Age was discovered to be highly correlated with class year; therefore, to maintain parsimonious data, age was left out of the analysis. With regards to GPA, $28(31 \%)$ of the participants reported a GPA in the 4.0-3.5 range, $32(35 \%)$ in the 3.5-3.0 range, $21(23 \%)$ in the 3.0-2.5 range, $5(6 \%)$ in the 2.5-2.0 range, $1(1 \%)$ reported a GPA below 2.0., and $4(4 \%)$ did not report GPA.

\section{Descriptive Data}

A series of one-way ANOVAs were conducted to assess for possible sex and class year differences on all predictor and criterion variables. These preliminary analyses were conducted as a justification for collapsing the different sample sub-groups. Using a probability level of .05, no significant mean differences were found for sex or class year. However, there was a marginally significant difference found by class year, as class year was found to be marginally related to academic self-concept $(p=.070)$ and social desirability $(p=.055)$. Nevertheless, due to the overall lack of significant differences amongst the studied demographic variables, the survey responses can be collapsed and analyzed as one group. A descriptive summary of the obtained 
means, standard deviations, and score ranges for each of the variables of interest is provided in Table 1.

TABLE 1

Means, Standard Deviations, and Ranges for all Variables

\begin{tabular}{|c|c|c|c|c|}
\hline Variable & Mean & $S D$ & $\begin{array}{c}\text { Potential } \\
\text { Range }\end{array}$ & $\begin{array}{c}\text { Obtained } \\
\text { Range }\end{array}$ \\
\hline MEIM & 62.98 & 7.33 & $20.00-80.00$ & $39.00-79.00$ \\
\hline Ethnic Identity & 42.33 & 6.67 & $14.00-56.00$ & $17.00-55.00$ \\
\hline Other Group Orientation & 20.65 & 2.21 & $6.00-24.00$ & $16.00-24.00$ \\
\hline ASCS & 117.67 & 16.39 & $40.00-160.00$ & $76.00-154.00$ \\
\hline RSES & 21.47 & 5.11 & $0.00-30.00$ & $9.00-30.00$ \\
\hline Grade Point Average & 2.93 & 0.95 & $0.00-4.00$ & $0.00-4.00$ \\
\hline Marlowe-Crowne: Form C & 7.18 & 2.68 & $0.00-13.00$ & $1.00-13.00$ \\
\hline
\end{tabular}

NOTE: MEIM = Multigroup Ethnic Identity Measure, ASCS $=$ Academic Self-Concept Scale, RSES = Rosenberg Self-Esteem Scale

Reliability coefficients were determined for the MEIM sub-factors and the ASCS inventory. The internal consistency reliability for Other Group Orientation (OGO) and Ethnic Identity (EI) was .74 and .90, respectively. The Cronbach's alpha for the ASCS inventory was found to be .90 .

The MEIM factor scores (i.e., EI \& OGO scores) were discovered to vary considerably in terms of their relationship to academic self-concept and self-esteem. Other Group Orientation (OGO) scores were determined to have a significant positive relationship $(p<.01)$ with both academic self-concept and self-esteem; however, OGO scores were not significantly correlated with GPA. Conversely, Ethnic Identity (EI) scores were not significantly correlated with self-esteem, academic self-concept, or GPA. Academic Self-Concept Scale (ASCS) scores were found to have a significant positive relationship ( $p<.01)$ with GPA, self-esteem (i.e., RSES scores), and OGO. Using a slightly weaker probability value of $.05, \mathrm{MC}-\mathrm{C}$ scores were found to 
be positively correlated with both OGO and ASCS scores. A correlation matrix for all research variables is presented in Table 2 .

TABLE 2

Correlation Coefficients for Variables

\begin{tabular}{|c|c|c|c|c|c|c|c|}
\hline & MEIM & $\begin{array}{l}\text { Ethnic } \\
\text { Identity }\end{array}$ & $\begin{array}{c}\text { Other } \\
\text { Group } \\
\text { Orientation }\end{array}$ & $A S C S$ & $R S E S$ & $\begin{array}{c}\text { Grade } \\
\text { Point } \\
\text { Average }\end{array}$ & $M C-C$ \\
\hline MEIM & -- & $.9543 * *$ & $.4346^{* *}$ & .0647 & .1933 & .0540 & .0748 \\
\hline Ethnic Identity & & -- & .1457 & -.0279 & .1043 & .0335 & .0135 \\
\hline Other Group Orientation & & & -- & $.2982 * *$ & $.3256^{* *}$ & .1468 & $.2187 *$ \\
\hline ASCS & & & & -- & $.4289 * *$ & $.4154 * *$ & $.2358^{*}$ \\
\hline RSES & & & & & -- & .0549 & .0954 \\
\hline Grade Point Average & & & & & & -- & .0415 \\
\hline MC-C & & & & & & & -- \\
\hline
\end{tabular}

NOTE: MEIM = Multigroup Ethnic Identity Measure, ASCS = Academic Self-Concept Scale, RSES = Rosenberg Self-Esteem Scale, MC-C = Marlowe-Crowne Social Desirability Scale: Short Form C $* p<.05 . * * p .01$ (two-tailed)

TABLE 3

Summary of Simultaneous Regression Analysis for Grade Point Average

\begin{tabular}{lccc}
\hline \hline Variable & $\beta$ & $t$ & $p<$ \\
\hline Ethnic Identity (EI) & .061 & .608 & .545 \\
Other Group Orientation (OGO) & -.044 & -.405 & .686 \\
ASCS & .506 & 4.436 & $.000^{*}$ \\
RSES & -.166 & -1.464 & .147 \\
\hline
\end{tabular}

NOTE: ASCS = Academic Self-Concept Scale, RSES = Rosenberg Self-Esteem Scale, ${ }^{*} p<.01$.

Because an additional purpose of this dissertation was to test a model that would predict GPA, a simultaneous multiple regression equation was calculated with ASCS scores, RSES scores, and MEIM factor scores as independent predictor variables. A forced-entry method was chosen over a step-wise method to examine the combined influence of the variables of interest (see Table 3). The best predictor of GPA was academic self-concept as measured by the ASCS. 
Self-esteem as measured by the RSES was not found to be predictive of GPA in the current sample. Similarly, EI and OGO (as measured by the MEIM) were both determined not to be statistically significant in terms of their relationship to GPA. Collectively, the independent variables accounted for $20 \%$ of the variance in GPA scores, as the cumulative $\mathrm{R}^{2}=.20, p<.01$. Academic self-concept accounted for the vast majority of the variance $\left(\mathrm{R}^{2}=.17\right)$, and had a positive relationship with GPA. Thus, in the current sample the higher the ASCS, the higher the GPA.

An evaluation of the ASCS eight factor structure (i.e., the factor structure unique to African American populations) as identified by Cokley et al. (2003), was applied to the current data. Data from African American students attending several HBCUs ( $n=396)$, as collected by Cokley et al. (2003), was compared to the data resultant from the current study of African American students at a PWI ( $n=91)$. In the present study, The Rewarded Efforts (RE) factor had the highest mean value $(M=3.48)$ and Study Habits $(\mathrm{SH})$ had the lowest mean value $(M=2.34)$. Descriptive statistics comparing the ASCS eight factor structure based on racial composition of the institution (i.e., HBCU vs. PWI) are provided in Table 4. 
TABLE 4

Academic Self-Concept Subscales for African American

Students by Racial Composition of Institution

\begin{tabular}{|c|c|c|c|c|}
\hline & $\begin{array}{l}\text { Summed } \\
\text { Scale M }\end{array}$ & $\begin{array}{c}\text { Average } \\
\text { Item } M\end{array}$ & $S D$ & $\begin{array}{c}\text { Number of } \\
\text { Items }\end{array}$ \\
\hline \multicolumn{5}{|l|}{ HBCU Setting* $(n=396)$} \\
\hline Study Habits & 10.50 & 2.10 & 3.09 & 5 \\
\hline Self-Confidence in Academics & 10.95 & 2.74 & 2.13 & 4 \\
\hline Satisfaction with Grades & 5.81 & 2.90 & 1.33 & 2 \\
\hline Self-Doubt Regarding Ability & 14.75 & 2.95 & 2.66 & 5 \\
\hline Evaluation of Academic Ability & 9.46 & 3.15 & 1.48 & 3 \\
\hline Discouragement About School & 6.35 & 3.18 & 1.61 & 3 \\
\hline Negative Performance Expectations & 6.60 & 3.30 & 1.39 & 2 \\
\hline Rewarded Efforts & 10.42 & 3.47 & 1.58 & 3 \\
\hline \multicolumn{5}{|l|}{ PWI Setting $(n=91)$} \\
\hline Study Habits & 11.68 & 2.34 & 3.08 & 5 \\
\hline Self-Confidence in Academics & 10.63 & 2.66 & 2.06 & 4 \\
\hline Satisfaction with Grades & 6.07 & 3.04 & 1.23 & 2 \\
\hline Self-Doubt Regarding Ability & 14.81 & 2.96 & 2.44 & 5 \\
\hline Evaluation of Academic Ability & 9.57 & 3.19 & 1.27 & 3 \\
\hline Discouragement About School & 8.92 & 2.97 & 2.32 & 3 \\
\hline Negative Performance Expectations & 6.88 & 3.44 & 1.41 & 2 \\
\hline Rewarded Efforts & 10.44 & 3.48 & 1.38 & 3 \\
\hline
\end{tabular}

NOTE: $\mathrm{HBCU}=$ Historically Black College and University, PWI $=$ Predominantly White Institution *Data taken from Cokley, Komarraju, King, Cunningham, \& Muhammad (2003).

\section{Test Descriptions and Data Characteristics}

The selection of statistical tests used to analyze the data obtained in the present study was done in accordance with the particular assumptions posed by each separate analysis, as determined by SPSS statistical software. For example, the incorporation of a one-way ANOVA to assess for sex and class year differences on all predictor and criterion variables was deemed appropriate due to a confirmation of the following assumptions: 1) Each sample served as an independent random sample; 2) The distribution of response variables followed a normal distribution pattern; and, 3) The population variances were equal across responses for the group levels. 
With regards to the use of a Pearson correlation coefficient with the current data, each set of compared variables were measured at the interval or ratio level (i.e., they were continuous), there was a determined linear relationship between the two variables, no significant outliers were detected in the present sample, and the measured variables followed an approximate normal distribution pattern. Therefore, the necessary assumptions were met accordingly in order to interpret the acquired data by way of a Pearson correlation coefficient.

A linear regression is the next step following a correlation, and it is the preferred test to use when the goal is to predict the value of one variable based on the value of another variable. Therefore, the data collected from the current sample was determined to have passed the following six assumptions in order to justify the incorporation of a regression analysis. First, all compared variables were measured at the continuous level (i.e., they were either interval or ratio variables). Next, it was determined that there was indeed a linear relationship between compared variables studied in the present analysis, as well as an absence of significant outliers. The fourth assumption for a linear regression asserts that one should have independence of observations. This was assessed in the current sample by way of a Durbin-Watson statistic run using SPSS statistical software. The value of the Durbin-Watson statistic ranges from 0 to 4 , with a value close to 0 being indicative of a strong positive correlation, while a value closer to 4 indicates a strong negative correlation. For the present study, the value of Durbin-Watson was 1.920, approximately equal to 2, indicating no serial correlation. Next, assumption five for the application of a linear regression relates to the homoscedasticity of the data. In the present data analysis, this assumption was also passed, as it was determined that the variances along the line of best fit remained similar as you move along the line. Finally, the sixth assumption of a linear regression model relates to the residuals (i.e., errors) of the regression line. It was determined via 
a histogram of the current data (with a super-imposed normal curve) that the residuals followed an approximate normal distribution pattern; thus, given the present study's findings it was confirmed that a linear regression model was indeed an appropriate and interpretable test.

\section{Hypotheses}

Hypothesis I. The initial hypothesis of the present study assumed that African American students at a PWI with higher MEIM factor scores would report higher RSES scores than African American students with lower MEIM factor scores. This hypothesis was partially supported based on the current findings determined by a way of a Pearson correlation coefficient. The OGO factor scores were found to be positively correlated with RSES scores $r=$ $.4289, p<.01$; however, the EI factor scores (although displaying a positive correlation) were not significantly correlated with RSES scores as $r=.1043$.

Hypothesis II. The second hypothesis asserted the assumption that African American students at a PWI with higher MEIM factor scores would report higher ASCS scores than African American students with lower MEIM factor scores. This hypothesis, along with the first, received only partial support, and this evidence was also based on findings determined by the calculation of a Pearson correlation coefficient. Similar to the first hypothesis, the OGO factor scores were found to be positively correlated with ASCS scores $r=.3256, p<.01$; however, the EI factor scores (displaying a slight negative correlation) were not significantly correlated with RSES scores as $r=-.0279$.

Hypothesis III. The third and final hypothesis predicted that African American students at a PWI with higher MEIM factor scores and higher ASCS scores would report higher GPA's than African American students with lower MEIM factor scores and lower ASCS scores. Again, this hypothesis received only partial support as evidenced by the results of the regression 
analysis applied to the present data. Academic Self-Concept Scale scores were predictive of GPA in the current sample $\left(R^{2}=.17, p<.01\right)$; however, MEIM scores were neither significantly correlated with, nor predictive of GPA in the current sample. In terms of the predictive value of EI and OGO with regard to GPA, the regression analysis determined p-values of .545 and .686, respectively; both well beyond the range of significance for predictability. 


\section{CHAPTER V \\ DISCUSSION}

The purpose of this dissertation was to explore the psychosocial impact of several factors, both dispositional (e.g., ethnic identity, self-esteem, and academic self-concept) and contextual (e.g., racial composition of the institution), thought to influence the academic experience of African American college students. As such, the following research questions were examined:

- Are the self-esteem and academic self-concept of African American college students at a PWI related to individual variations in ethnic identity?

- What is the relationship between ethnic identity, self-esteem, academic self-concept, and grade point average (GPA); and, what is the best predictor model for GPA?

The results as they relate to the research questions and relevant literature will be presented in this section. Limitations of the study and implications for future research and practice will also be discussed.

\section{Multigroup Ethnic Identity Measure and ASCS, RSES, and GPA}

Of the two Multigroup Ethnic Identity Measure (MEIM) sub-factors, Other Group Orientation (OGO) scores were significantly correlated with Academic Self-Concept Scale (ASCS) and Rosenberg Self-Esteem Scale (RSES) scores; however, Ethnic Identity (EI) scores were not significantly correlated with either ASCS or RSES scores. The finding that EI scores were not significantly correlated with either ASCS or RSES scores was consistent with the findings of Witherspoon et al. (1997); however, this finding was inconsistent with the proposed hypotheses I and II (as stated in Chapter IV).

Previous research on African American students had largely shown that self-esteem and ethnic identity were significantly related variables (Lockett \& Harrell, 2003; Phinney \& Chavira, 
1992; Phinney, 1996; Phinney, Cantu, \& Kurtz, 1997); therefore, the lack of significance between with EI and RSES scores in the current sample was somewhat surprising. Likewise, the majority of extant research on the academic self-concept of African American students had revealed a significant positive correlation with ethnic identity achievement (Awad, 2007; Cokley, 2000; Cokley, 2002; Johnson \& Arbona, 2006; Schmader, Major, \& Gramzow, 2001); thus, the observed non-significance between EI scores and ASCS was also unexpected. It is important to recognize that the findings of the present investigation were based on African American undergraduate and graduate students, as opposed to the previously cited research which was based largely on undergraduate students.

\section{Hypothesis I.}

In response to the significant positive correlation observed between OGO scores and RSES scores, this finding was consistent with the proposed hypothesis I, and challenges the Witherspoon et al. (1997) observation that racial identity attitudes were unrelated to selfesteem. Likewise, the argument offered by Hughes and Demo (1989), which posits that social contact with White Americans is generally unimportant to the self-esteem of African Americans, is also called into question based on the present findings.

\section{Hypothesis II.}

The positive correlation detected between OGO scores and ASCS scores in the current investigation was consistent with the proposed hypothesis II, and is comparable to the research findings of Cole and Yip (2008). Cole and Yip investigated a construct they labeled out-group comfort (a construct partially derived from items contained in the OGO factor of the MEIM), and out-group comfort was determined to be positively correlated with academic self-concept. Therefore, this suggested likeness in construct infers that higher OGO scores may function as a 
buffer for African American students at a PWI, similar to the role of out-group comfort as observed by Cole and Yip (2008). Basically, higher OGO may allow African American students greater social ease with dominant group members and then enhance the potential for improved academic outcomes in predominantly White institutional settings.

Rosenberg Self-Esteem scores were shown to be positively correlated with ASCS scores in the current sample. As self-esteem increased, so did academic self-concept; likewise, as selfesteem decreased, so did academic self-concept. Unfortunately, it is not possible to determine causation from this correlation. Although not statistically significant at a probability value of .01 , the MC-C scores were shown to be positively correlated with both ASCS scores and OGO scores when using a probability value of .05 . In other words, the trend suggested that as social desirability increased for African American students at a PWI, so too did academic self-concept and other group orientation. However, as was the case with self-esteem, it is not possible to determine causation from these correlations.

\section{Hypothesis III.}

With regards to hypothesis III, ASCS scores were significantly correlated with and predictive of GPA in the current sample; however, MEIM scores (i.e., EI and OGO scores) were neither significantly correlated with, nor predictive of GPA. Thus, hypothesis III received only partial support as determined by the relative significance of academic self-concept. In the present investigation, the best predictor of GPA for African American students at a PWI was academic self-concept as measured by the ASCS. Academic self-concept accounted for $17 \%$ of the variance in GPA scores, and had a positive relationship with GPA.

Therefore, in the current sample of African American college students at a PWI the higher the ASCS, the higher the GPA. This is contrary to the findings of Witherspoon et al. 
(1997) wherein GPAs for African American high-school students were best predicted by immersion racial identity attitudes and academic self-concept. Interestingly, in the Witherspoon et al. (1997) study none of the various racial identity attitudes were significantly correlated with academic self-concept or self-esteem, whereas the present investigation revealed the OGO sub-factor of ethnic identity to be significantly correlated with both academic self-concept and self-esteem.

\section{Comparisons Based on Institutional Context}

Previous researchers exploring differences in institutional context for African American college student populations found that African American students at HBCUs tended to have generally higher academic self-concepts when compared to African American students attending PWIs (Allen, 1992; Cokley, 2002; Cokley \& Moore, 2007; Gerardi, 1990). Allen (1992) studied the college experiences of 953 African American undergraduate students who attended HBCUs and 1,578 who attended PWIs. He discovered African American students attending PWIs reported lower GPAs and academic self-concepts, as well as lower levels of social involvement. Furthermore, his results suggest that outcomes were influenced by the immediate social context, while interpersonal relationships bridged individual disposition and institutional context.

In the present investigation, the positive correlation observed between ASCS scores and OGO scores conveys the notion that higher levels of social involvement with out-group members likely contributes to better academic outcomes and higher academic self-concepts for African American students at a PWI. Therefore, African American students attending a PWI may likely benefit from social involvement with out-group members, as the potentially negative influence posed by a predominantly White social and institutional context appears to be lessened. In other 
words, a greater degree of OGO could provide a valuable advantage to African American students attending PWIs.

For the purpose of comparison, the ASCS eight factor structure (i.e., the factor structure unique to African American populations) as identified by Cokley et al. (2003), was applied to the collected data. Descriptive statistics comparing the mean value of items (as determined by the ASCS eight factor structure) revealed some intriguing differences between African American college students based on institutional context. For example, when comparing averaged item means (see Table 4) African American students from this study at a PWI reported better perceived study habits, greater satisfaction with grades, and less negative performance expectation than African American students at HBCUs as measured by Cokley et al. (2003). This finding was somewhat surprising, as social identity theorists (e.g., Negy et al., 2003; Yee \& Brown, 1992) would likely argue that a PWI setting might inhibit the development of such factors. Conversely, African American students at an HBCU reported less discouragement about school than students at a PWI, as lower scores on the Discouragement About School (DAS) factor reflected greater discouragement about school (see Table 4). The observed difference on the DAS factor was consistent with social identity theory in that the African American students in a predominantly White social and institutional context reported, on average, higher levels of discouragement about school than African American students at an HBCU.

\section{Limitations \& Strengths of the Present Study}

The participants in this study were enrolled at a single PWI in the mid-Atlantic region of the United States; this particular environment may have had an effect on the results found. The institution of higher education sampled in this dissertation exists in a largely rural area, within a largely rural and predominantly White state; thus, it is likely that many of the participants in the 
current study were reared in social environments similar to that of a PWI setting. Therefore, these results may have limited generalizability to other institutional settings and geographic regions. What is more, although the current sample size $(n=91)$ was adequate (per a power analysis set to detect medium effect size at a .05 alpha level), it did not allow for enough variance to be detected amongst the variables of interest. For example, only six participants endorsed a GPA below 2.5, which contributed to a significantly imbalanced representation of range in GPA, thereby compromising the value of the prediction model used to address hypothesis III. In addition, there was limited representation with regards to sexual diversity in the studied sample as only $23(25 \%)$ of respondents identified as male and $59(65 \%)$ female.

The manner in which GPA was gathered posed additional limitations to the present analyses. Grade point average was determined by responses on the Demographic Questionnaire (see Appendix E) and was identified by selecting a range; however, in order to analyze the current data, GPA needed to be a continuous variable. The GPA ranges were translated to a scale where they could be used as a continuous variable (see GPA Range Translation Scale in Appendix E), but this translation likely compromised the data to some degree. Therefore, in future studies wherein GPA is being used for comparative purposes, it is recommended to capture GPA as a continuous variable rather than a range.

In the current sample, the overall diversity amongst majors at both the undergraduate and graduate level (see Appendix G) posed limitations on the ability to infer differences based on major. Likewise, the observed high correlation between class year and age limited the ability to differentiate scores based on either of these variables. Again, it is likely that a larger sample size may have allowed for detection of such differences (e.g., differences based on major, graduate/undergraduate status, and class year). 
As a primary limitation of the present study, it is important to recognize that correlation does not imply causation. A substantial number of the reported findings in the present study are offered by way of a Pearson correlation coefficient. Furthermore, the OGO factor (as measured by the MEIM) only constitutes a portion of one's ethnic identity; therefore, overall ethnic identity achievement cannot be generalized from an OGO score. Consequently, all findings which indicated significance relative to the OGO factor score must be considered in a limited fashion. In future studies, it is possible that out-group comfort (as studied by Cole \& Yip, 2008) might better explain the relationship between self-esteem, academic self-concept and outcomes for African American students at PWIs.

The issue of racial/ethnic self-identification posed another challenge in data interpretation for the present investigation. For example, 77 participants $(85 \%)$ identified as Black or African American (i.e., a singular race/ethnicity), whereas the remaining 14 participants (15\%) reported a multi-racial/ethnic identity of which Black or African American was a portion. As such, differences in the race/ethnicity of participants (i.e., participants who endorsed multiple racial/ethnic identities as opposed to a singular race/ethnicity) were unable to be determined due to the limited diversity and overall lack of respondents in this study. In terms of general identification as Black or African American, the present study did not differentiate international students of African descent from U.S. born students of African descent. The distinctive cultural differences between international born and U.S. born students could indeed contribute to significant variation in their endorsements; therefore, future studies on African American students should assess for differences in country of origin as well as multiple racial/ethnic identities. 
Limitations in demographic variation and overall sample size precluded the incorporation of a hierarchical regression analysis, as the ability to parse out the effect of control variables was thereby compromised. For a hierarchical regression analysis, the preferred minimum subject-tovariable ratio is 15 to 1 (Stevens, 2009). The present study had a subject ( $n=91)$ to variable (9) ratio of 10.1 to 1 , which implies a larger subject-to-variable ratio would be required to attain the necessary predictive power for a hierarchical regression analysis.

Regardless of the aforementioned limitations, the present study was not without its respective strengths. For example, each of the incorporated statistical analyses was found to pass the required assumption tests, which in turn offers strength to the reliability and validity of the observed findings. Furthermore, the procedural inconsistencies identified in the Witherspoon et al., (1997) study were avoided in the present study as all data was collected in the same fashion (i.e., submitted electronically via Qualtrics) and time-frame (i.e., within a 2-week window near the end of the Fall semester). The inclusion of the RSES as the measure of selfesteem (as opposed to the POI) also contributed to the strength of the study, as the utilization of the RSES revealed significant findings that may have gone undetected with a less reliable and valid measure.

Lastly, a principal strength of the present study was that by investigating the sub-factors of the MEIM (i.e., EI and OGO), it was possible to detect significant differences within the construct of ethnic identity itself. Previous researchers studying the construct of ethnic identity, by and large, did not differentiate the sub-factors of the MEIM in their investigations; thus the present study is unique in terms of its ability to clarify the relative significance of the EI and OGO sub-factors of ethnic identity achievement, specifically for African American students within a PWI setting. 


\section{Implications \& Suggestions for Future Research and Practice}

Extant research, considered together with the present findings, has demonstrated that African American students who endorse an achieved ethnic identity (i.e., higher scores on the MEIM) are typically found to have more comfortable and adaptive social interactions, which translates to healthier overall functioning (Allen, 1992; Cokley, 2002; Cokley, 2005; Phinney, Jacoby, \& Silva, 2007). In addition, individuals with a more achieved ethnic identity have a tendency to engage their environment in a more frequent and assertive manner (Rowley, Sellers, Chavous, \& Smith, 1998); however, in the present study healthy engagement within a PWI setting appears to be more attributable to the influence of the OGO sub-factor rather than the EI sub-factor of ethnic identity achievement. More importantly, these findings suggest that African American students at PWIs may benefit from contact and involvement with other students, regardless of the student's race/ethnicity, as this seems to be advantageous to the development of an achieved ethnic identity.

Earlier research conducted by Moeller (1994), informed parents and educators that efforts to improve academic performance in children have often centered on raising their selfesteem with programs like "Project Follow Through," a federal program with a self-esteem component, to assist Head Start children in Grades 1 through 3. He concluded, however, that such efforts are misplaced because the research indicates that increased self-esteem rarely leads to improved academic performance. The present study supports the conclusions drawn by Moeller (1994), and challenges the Frerichs (1971) study which conveyed a significant positive relationship between self-esteem and school success.

Researchers in the social sciences have discovered that African American students have levels of self-esteem and academic self-concepts that are, on average, equal to or higher than 
those of White students (Awad, 2007; Cokley, 2000; Cokley et al., 2003; Lockett \& Harrell, 2003; Negy et al., 2003; Phinney, Cantu, \& Kurtz, 1997; Schmader, Major, \& Gramzow, 2002); and, the self-esteem of African American students is also unrelated to their grades (Cokley, 2000; Cokley et al., 2003). The present study furthers the notion that self-esteem is not a significant variable in terms of its predictive influence on GPA. Thus, psychologists and educators dealing with academic problems of African American students should likely place less emphasis on self-esteem, when potentially more productive efforts (e.g., focusing on the fear of confirming negative stereotypes, and/or targeting the elimination of campus racism and discrimination) could be pursued. It is also recommended that psychologists, particularly those focused on helping African American college students at PWIs, be supportive in the development of positive relationships with faculty, as this has been shown to be a valuable resource for the African American students who established such relationships (Cokley, 2000; Cokley, 2002; Cokley \& Moore, 2007).

Although an African American student may de-identify with academics (Steele, 1997), his/her self-esteem is likely to remain high, as self-esteem is no longer contingent upon and/or related to the student's academic performance (Cokley \& Moore, 2007). In other words, the experience of psychological disengagement has varying effects on the ethnic identity and academic self-concept of African American college students, and some of this difference is purportedly influenced by sex (Cokley \& Moore, 2007). However, in the present investigation there were no significant differences determined on the basis of sex.

\section{Summary and Conclusion}

This study provides only a snapshot of students' development. Many questions remain about how African American students develop ethnic identity and whether ethnic identity is 
related to their self-esteem, self-concept, or academic outcomes. Phinney, Ferguson, and Tate (1997) found that ethnic diversity outside of school contexts predicted out-group friendships among minority high school students, which in turn predicted positive out-group attitudes. This suggests that residential segregation (i.e., living in a predominantly Black or White dorm and/or neighborhood) may be an important obstacle to the development of ethnic identity. Moreover, in a sample of college students, Levin et al. (2003) showed that low intergroup anxiety and positive out-group contact may have a positive feedback loop such that low intergroup anxiety predicts out-group friendships which in turn predict low intergroup anxiety over time. These studies, considered together with present research, illustrate how critically important it is to situate our understanding of the precursors and consequences of ethnic identity, namely the OGO factor.

The present study did not examine changes in ethnic identity over time; however, future research should investigate both the precursors and consequences of ethnic identity and the factors that might enhance its development, such as participation in social networks or extracurricular activities. This research could form the basis for interventions (e.g., orientation programs) to support the development of ethnic identity among African American students at PWIs (see, e.g., Pinel et al., 2005).

In the current sociopolitical landscape, there is increasing interest in the educational benefits of diversity (Gurin, Dey, Hurtado, \& Gurin, 2002; Gurin, Hagda, \& Lopez, 2004; Pascarella et al., 2014). This conversation must include attention to the experiences of minority group members within racially/ethnically imbalanced and diverse collegiate settings. The current findings suggest that African American students at a PWI with a greater degree of other group orientation are poised to take greater advantage of the benefits diversity may offer. This study demonstrates that a social and educational environment composed primarily of out-group 
members (e.g., a PWI) may not necessarily impede functioning of African American college students any differently than a racially/ethnically balanced environment (e.g., an HBCU). More importantly, it is possible given the current findings that a PWI environment could enhance academic confidence and overall well-being for students with a more achieved ethnic identity. It is therefore the responsibility of psychologists, as well as faculty and college administrators to deepen our understanding of the factors that may influence ethnic identity achievement, and to subsequently institute policies and practices that promote a healthier social and institutional environment.

In conclusion, this investigation has found that there are indeed within group differences in the ethnic identity achievement, self-esteem and academic self-concept of African American college students at a PWI that may be related to the various ways that students perform in school. It is hoped that with future research focused on the development of psychoeducational interventions (specifically those aimed at helping African American students at PWIs), psychologists, educators, and policy makers alike will thereby increase their potential to cultivate a more positive interpersonal and academic experience for all students. 


\section{REFERENCES}

Allen, W. R. (1992). The color of success: African American college student outcomes at predominantly White and historically Black public colleges and universities. Harvard Educational Review, 62, 26-44.

American Council on Education (1990). Minorities in higher education. Washington, D. C.: Office of Minority Concerns.

American Psychological Association. (2003). Guidelines on multicultural education, training, research, practice, and organizational change for psychologists. American Psychologist, 58, 377-402.

Andrews, P., \& Meyer, R. G. (2003). Marlowe-Crowne Social Desirability Scale and short form c: Forensic norms. Journal of Clinical Psychology, 59, 483-492.

Arminio, J.L., Carter, S., Jones, S.E., Kruger, K., Lucas, N., Washington, J., Young, N., \& Scott, A. (2000). Leadership experiences of students of color. NASPA Journal, 37, 496510.

Arriola, K. R, \& Cole, E. R. (2001). Framing the affirmative-action debate: Attitudes toward outgroup members and White identity. Journal of Applied Social Psychology, 31, 24622483.

Arroyo, C. G., \& Zigler, E. (1995). Racial identity, academic achievement and the psychological well-being of economically disadvantaged adolescents. Journal of Personality and Social Psychology, 69, 903-914.

Avery, D. R., Tonidandel, S., Thomas, K. M., Johnson, C. D., \& Mack, D. A. (2007). Assessing the multigroup ethnic identity measure for measurement equivalence across racial and ethnic groups. Educational \& Psychological Measurement, 67, 877-888. 
Awad, G. (2007). The role of racial identity, academic self-concept, and self-esteem in the prediction of academic outcomes. Journal of Black Psychology, 33, 188-207.

Baldwin, J. A., Brown, R., \& Rackley, R. (1990). Some socio-behavioral correlates of African self-consciousness in African American college students. The Journal of Black Psychology, 17, 1-17.

Ballard, R. (1992). Short forms of the Marlowe-Crowne Social Desirability Scale. Psychological Reports, 71, 1155-1160.

Beretvas, S. N., Meyers, J. L., \& Leite, W. L. (2002). A reliability generalization study of the Marlowe-Crowne Social Desirability Scale.” Educational and Psychological Measurements, 62, 570-589.

Branscombe, N. R., Schmitt, M. T., \& Harvey, R. D. (1999). Perceiving pervasive discrimination among African Americans: Implications for group identification and well-being. Journal of Personality \& Social Psychology, 77, 135-149.

Brown v. Board of Education of Topeka, 347 U.S. 483 (1954).

Brown, R. T., Reynolds C. R., \& Whitaker J. S. (1999) Bias in mental testing since "Bias in Mental Testing”. School Psychology Quarterly, 14, 1999, 208-238.

Burkard, A. W., Medler, B. R., \& Boticki, M. A. (2001). Prejudice and racism: Challenges and progress in measurement. In J. G. Ponterotto, J. Casas, L. A. Suzuki, C. M. Alexander (Eds.). Handbook of multicultural counseling (2 ${ }^{\text {nd }}$ ed.), (pp. 457-481). Thousand Oaks CA: Sage Publications, Inc.

Cardemil, E. V., \& Battle, C. L. (2003). Guess who's coming to therapy? Getting comfortable with conversations about race and ethnicity in psychotherapy. Professional Psychology: Research and Practice, 34, 278-286. 
Chavous, T. M. (2000). The relationship among racial identity, perceived ethnic fit, and organizational involvement for African American students at a predominantly White university. Journal of Black Psychology, 26, 79-100.

Chavous, T. M., Bernat, D. H., Schmeelk-Cone, K., Caldwell, C. H., Kohn-Wood, L., \& Zimmerman, M. A. (2003). Racial identity and academic achievement among African American adolescents. Child Development, 74, 1076-1090.

Clark, R, Anderson, N. B., Clark, V. R., \& Williams, D. R. (1999). Racism as a stressor for African-Americans. American Psychologist, 54, 805-816.

Cohen, J. (1992). A power primer. Psychological Bulletin, 112, 155-159.

Cole, E. R., \& Yip, T. (2008). Using out-group comfort to predict Black students' college experience. Cultural Diversity and Ethnic Minority Psychology, 14, 57-66.

Cokley, K. (2000). An investigation of academic self-concept and its relationship to academic achievement in African American college students. Journal of Black Psychology, 26, 148-164.

Cokley, K. (2002). Ethnicity, gender, and academic self-concept: A preliminary examination of academic disidentification and implications for psychologists. Cultural Diversity and Ethnic Minority Psychology, 8, 378-388.

Cokley, K., Komarraju, M., King, A., Cunningham, D., \& Muhammad, G. (2003). Ethnic differences in the measurement of academic self-concept in a sample of African American and European American college students. Education and Psychological Measurement, 63, 707-722. 
Cokley, K. (2005). Racialized identity, ethnic identity, and Afrocentric values: Conceptual and methodological challenges in understanding African American identity. Journal of Counseling Psychology, 52, 517-526.

Cokley, K. (2007). Critical issues in the measurement of ethnic and racial identity: A referendum on the state of the field. Journal of Counseling Psychology, 54, 224-234.

Cokley, K., \& Moore, P. (2007). Moderating and mediating effects of gender and psychological disengagement on the academic achievement of Black/African college students. Journal of Black Psychology, 33, 169-187.

Corwyn, R. F. (2000). The factor structure of global self-esteem among adolescents and adults. Journal of Research in Personality, 34, 357-379

Cross, W. E. (1971). The Negro to Black Experience. The East Publishing Company.

Cross, W. E. (1991). Shades of Black: Diversity in African American identity. Philadelphia: Temple Press.

Cross, W. E., \& Strauss, L. (1998). The everyday functions of African American identity. In J. K. Swim, L Stangor (Eds.), Prejudice: The target's perspective (p.267-279). San Diego, CA: Academic Press.

Crowne, D. P., \& Marlowe, D. (1960). A New Scale of Social Desirability Independent of Psychopathology. Journal of Consulting Psychology, 24, 349-354.

Davis, C., \& Cowles, M. (1989). Automated psychological testing. Method of administration, need for approval, and measures of anxiety. Education and Psychological Measurement, 49, 311-320. 
Dovidio, J. F., Gaertner, S. L., Kawakami K., \& Hodson, G. (2002). Why can’t we all just get along? Interpersonal biases and interracial distrust. Cultural Diversity and Ethnic Minority Psychology, 8, 88-102.

Dunbar, M., Ford, G., Hunt, K., \& Der, G. (2000). Question wording effects in the assessment of global self-esteem. European Journal of Psychological Assessment, 16, 13-19.

Easley, N. (1993). Black student retention at Colorado State University. Unpublished manuscript, Colorado State University at Fort Collins.

Erikson, E. (1968). Identity youth and crisis. New York: W. W. Norton.

Fischer, A. R., \& Moradi, B. (2001). Racial and ethnic identity: Recent developments and needed directions. In J. G. Ponterotto, J. M. Casas, L. A. Suzuki, \& C. M. Alexander (Eds.), Handbook of multicultural counseling (pp. 341-370). Thousand Oaks, CA: Sage.

Fleming, J. (1984). Blacks in college. San Francisco: Jossey-Bass.

Fleming, J. S., \& Courtney, B. E. (1984). The dimensionality of self-esteem: II. Hierarchical facet model for revised measurement scales. Journal of Personality and Social Psychology, 46, 404-421

Foley, D. (2005). Elusive prey: John Ogbu and the search for a grand theory of academic disengagement. International Journal of Qualitative Studies in Education, 18, 643-657.

Fordham, S., \& Ogbu, J. U. (1986). Black students' school success: Coping with the 'burden of acting White.'. The Urban Review, 18(3), 176-206.

Fowers, B. J., \& Davidov, B. J. (2006). The virtue of multiculturalism. American Psychologist, 61, 581-594.

Frerichs, A. H. (1971). Relationship of self-esteem of the disadvantaged to school success. The Journal of Negro Education, 40, 117-120. 
Fries-Britt, S., \& Griffin, K. A. (2007). The Black box: How high-achieving Blacks resist stereotypes about Black Americans. Journal of College Student Development, 48, 509524.

Gerardi, S. (1990). Academic self-concept as a predictor of academic success among minority and low-socioeconomic status students. Journal of College and Student Development, $31,402-407$.

Graham, S. (1997). Using attribution theory to understand academic motivation in African American youth. Educational Psychologist, 32, 21-34.

Greenberg, B., \& Weiss, P. (2012). Validation of a short form of the Marlowe-Crowne for use with law enforcement personnel. Journal of Police \& Criminal Psychology, 27, 123-128.

Gurin, P., Dey, E. L. Hurtado, S., \& Gurin, G. (2002). Diversity and higher education: Theory and impact on educational outcomes. Harvard Educational Review, 72, 330-366.

Gurin, P., Nagda, B. A., \& Lopez, G. E. (2004). The benefits of diversity in education for democratic citizenship. Journal of Social Issues, 60, 7-34.

Hatcher, J., \& Hall, L. A. (2009). Psychometric properties of the Rosenberg self-esteem scale in African American single mothers. Issues in Mental Health Nursing, 30, 70-77.

Helms, J. E. (1990). Black and White racial identity: Theory and research. Westport, CT: Greenwood.

Hendrix-Wright, B. (1981). Influences on self-esteem: Internal versus external control and racial group identification. Journal of Social and Behavioral Sciences, 27, 12-22.

Hughes, M., \& Demo, D. H. (1989). Self-perceptions of Black Americans: Self-esteem and personal efficacy. American Journal of Sociology, 95, 132-159.

Ivey, A. E., D’Andrea, M., Ivey, M. B., \& Morgan, L. S. (2002). Theories of Counseling 
and Psychotherapy: A Multicultural Perspective, $5^{\text {th }}$ ed. Boston, MA: Allyn \& Bacon.

Johnson, S. C., \& Arbona, C. (2006). The relation of ethnic identity, racial identity, and race-related stress among African American college students. Journal of College Student Development, 47, 495-507.

Koch, L. M., Gross, A. M., \& Kolts, R. (2001). Attitudes toward Black English and code switching. The Journal of Black Psychology, 27, 29-42.

Lent, R. W., Brown, S. D., \& Gore, P. A. (1997). Discriminate and predictive validity of academic self-concept, academic self-efficacy, and mathematics-specific self-efficacy. Journal of Counseling Psychology, 44, 307-315.

Levin, S., Van Laar, C., \& Sidanius, J. (2003). The effects of in-group and out-group friendships on ethnic attitudes in college: A longitudinal study. Group Processes and Intergroup Relations, 6, 76-92.

Lockett, C. T., \& Harrell, J. P. (2003). Racial identity, self-esteem, and academic achievement: Too much interpretation, too little supporting data. Journal of Black Psychology, 29, 325-336.

Loo, R., \& Thorpe, K. (2000). Confirmatory factor analyses of the full and short versions of the Marlowe-Crowne Social Desirability Scale. The Journal of Social Psychology, 140, 628635.

Marcia, J. E. (1980). Identity in adolescence. In J. Edelson (Ed.), Handbook of adolescent psychology (pp. 159-165, Chapter 5). New York: John Wiley.

Marks, K. M., Szalacha, L. A., Lamarre, M., Boyd, M. J., \& Garcia-Coll, C. (2007). Emerging ethnic identity and interethnic group social preferences in middle childhood: Findings 
from the Children of Immigrants Development in Context (CIDC) study. International Journal of Behavioral Development, 31, 501-513.

McCreary, M. L., Slavin, L. A., \& Berry, E. J. (1996). Predicting problem behavior and selfesteem among African American adolescents. Journal of Adolescent Research, 11, $216-234$.

Mickelson, R. A. (1990). The attitude-achievement paradox among Black adolescents. Sociology of Education, 63, 44-61.

Moeller, T. J. (1994). What research says about self-esteem and academic performance. Virginia Journal of Education, 87, 6-11.

Morin, R. (2001). Misperceptions cloud Whites' view of Blacks. Washington Post, July $11,2001$.

Myers, L. (1988). Understanding an Afrocentric worldview: Introduction to an optimal psychology. Dubuque, IA: Kendall/Hunt.

National Center for Education Statistics. (2012). Digest of Education Statistics, 2011. Postsecondary education (pp. 279-412, Chapter 3), Washington, DC: Author. National Center for Education Statistics. (2014). Enrollment in Postsecondary Institutions, Fall 2013; Financial Statistics, Fiscal Year 2013; and Employees in Postsecondary Institutions, Fall 2013 First Look (Provisional Data), Washington, DC: Author.

Negy, C., Shreve, T. L., Jensen, B. J., \& Uddin, N. (2003). Ethnic identity, self-esteem, and ethnocentrism: A study of social identity versus multicultural theory of development. Cultural Diversity and Ethnic Minority Psychology, 9, 333-344.

Ogbu, J. U. (1978). Minority Education and Caste: The American System in Cross-Cultural Perspective. San Diego, CA: Academic Press. 
Ogbu, J. U. (1981). Origins of human competence: A cultural-ecological perspective. Child Development, 52(2), 413-429.

Osborne, J. O. (1995). Academics, self-esteem, and race: A look at the underlying assumptions of the disidentification hypothesis. Personality and Social Psychology Bulletin, 21, 449455.

Palmer, R. T., \& Young, E. M. (2009). Determined to succeed: Salient factors that foster academic success for academically unprepared Black males at a Black college. Journal of College Student Retention, 10, 465-482.

Parham, T. A., \& Helms, J. E. (1981). The influence of Black students' racial identity attitudes on preference for counselor's race. Journal of Counseling Psychology, 28, 250-257.

Parham, T. A., \& Helms, J. E. (1985). Relation of racial identity attitudes to self-actualization and affective states of Black students. Journal of Counseling Psychology, 32, 431-440.

Pascarella, E. T., Martin, G. L., Hanson, J. M., Trolian, T. L., Gillig, B., \& Blaich, C. (2014). Effects of diversity experiences on critical thinking skills over 4 years of college. Journal Of College Student Development, 55(1), 86-92.

Paulhus, D. L. (2002). Socially desirable responding: The evolution of a construct. In H. I. Braun, D. N. Jackson, \& D. E. Wiley (Eds.), The role of constructs in psychological and education measurement (pp. 49-69). Mahwah, NJ: Erlbaum.

Phinney, J. S. (1989). Stages of ethnic identity development in minority group adolescents. The Journal of Early Adolescence, 9, 34-49.

Phinney, J. S. (1992). The multigroup ethnic identity measure: A new scale for use with diverse groups. Journal of Adolescent Research, 7, 156-176. 
Phinney, J. S. (1996). Understanding ethnic diversity: The role of ethnic identity. American Behavioral Scientist, 40, 143-152.

Phinney, J. S., (1996). When we talk about American ethnic groups, what do we mean? American Psychologist, 51, 918-927.

Phinney, J. S., Ferguson, D. L., \& Tate, J. D. (1997). Intergroup attitudes among ethnic minority adolescents: A causal model. Child Development, 68, 955-969.

Phinney, J. S., Cantu, C. L., \& Kurtz, D. A. (1997). Ethnic and American identity as predictors of self-esteem among African American, Latino, and White adolescents. Journal of Youth and Adolescence, 26I, 165-185.

Phinney, J. S., Jacoby, B., \& Silva, C. (2007). Positive intergroup attitudes: The role of ethnic identity. International Journal of Behavioral Development, 31, 478-490.

Pinel, E., Warner, L., \& Chua, P. (2005). Getting there is only half the battle: Stigma consciousness and maintaining diversity in higher education. Journal of Social Issues, 61, 481-506.

Reynolds, W. M., Ramirez, M. P., Magrina, A., \& Allen, J. E. (1980). Initial development and validation of the academic self-concept scale. Educational and Psychological Measurement, 1980, 1013-1016.

Reynolds, W. M. (1982). Development of reliable and valid short forms of the MarloweCrowne Social Desirability Scale. Journal of Clinical Psychology, 38, 119-125.

Reynolds, W. M. (1988). Measurement of academic self-concept in college students. Journal of Personality Assessment, 52, 223-240. 
Richardson, C. G., Ratner, P. A., \& Zumbo, B. D. (2009). Further support for multidimensionality within the Rosenberg self-esteem scale. Current Psychology, $28,98-114$.

Rosenberg, M. (1965). Society and the adolescent self-image. Princeton, NJ: Princeton University Press.

Rosenberg, M. (1979). Conceiving the self. New York: Basic.

Rowley, S. J., Sellers, R. M., Chavous, T. M., \& Smith, M. A. (1998). The relationship between racial identity and self-esteem in African American college and high school students. Journal of Personality and Social Psychology, 74, 715-724.

Schmader, T., Major, B., \& Gramzow, R. H. (2001). Coping with ethnic stereotypes in the academic domain: Perceived injustices and psychological disengagement. Journal of Social Issues, 57, 99-111.

Schmader, T., Major, B., \& Granzow, R. (2002). How African American college students protect their self-esteem. The Journal of Blacks in Higher Education, 35, 116-119.

Schmitt, D. P., \& Allik, J. (2005). Simultaneous administration of the Rosenberg Self-Esteem Scale in 53 nations: Exploring the universal and culture-specific features of global self-esteem. Journal of Personality and Social Psychology, 89, 623-642.

Seidner, S. S. (1982). Ethnicity, Language, and Power from a Psycholinguistic Perspective, Bruxelles: Centre de Recherche sur le Pluralinguisme.

Sellers, R. M., Rowley, S. A., Chavous, T. M., Shelton, J. N., \& Smith, M. A. (1997). Multidimensionality inventory of Black identity: A preliminary investigation of reliability and construct validity. Journal of Personality \& Social Psychology, $73,805-815$. 
Sellers, R. M., \& Shelton, J. (2003). The role of racial identity in perceived racial discrimination. Journal of Personality and Social Psychology, 84, 1079-1092

Sellers, R. M., Smith, M. A., Shelton, J. N., Rowley, S. A., \& Chavous, T. M. (1998). Multidimensional model of racial identity: A reconceptualization of African American identity racial identity. Personality \& Social Psychology Review, 2, 18-39.

Shelton, J. N. (2000). A reconceptualization of how we study issues of racial prejudice. Personality and Social Psychology Review, 4, 374-390.

Shostrom, E., (1963). Personal Orientation Inventory. San Diego, CA: Educational and Industrial Testing Service.

Silbert, E., \& Tippett, J. (1965). Self-esteem: Clinical assessment and measurement validation. Psychological Reports, 16, 1017-1071.

Smedley, A., \& Smedley, B. D. (2005). Race as biology is fiction, racism as a social problem is real: Anthropological and historical perspectives on the social construction of race. American Psychologist, 60, 16-26.

Smith, E. (2006). The strength-based counseling model. Counseling Psychologist, 34, 13-79. Steele, C. M. (1992, April). Race and the schooling of Black Americans. The Atlantic Monthly. pp. 68-77.

Steele, C. M., \& Aronson, J. (1995). Stereotype threat and the intellectual test performance of African Americans. Journal of Personality and Social Psychology, 69, 797-811.

Steele, C. M. (1997). A threat in the air: How stereotypes shape the intellectual identities and performance. American Psychologist, 52, 613-629.

Stevens, J. P. (2009). Applied Multivariate Statistics for the Social Sciences, $5^{\text {th }}$ Ed., New York: Taylor and Francis. 
Strahan, R., \& Gerbasi, K. C. (1972). Short, homogeneous versions of the Marlowe-Crowne Social Desirability Scale. Journal of Clinical Psychology, 28, 191-193.

Sue, D. W. (2004). Multicultural counseling and therapy (MCT). In J.A. Banks and C. Banks (Eds.) Handbook of Research on Multicultural Education, 2nd Ed., 813-827., San Francisco: Jossey-Bass.

Sue, D. W., Capodilupo, C. M., Torino, G. C., Bucceri, J. M., Holder, A. M., Nadal, K. L., \& Esquilin, M. (2007). Racial microaggressions in everyday life: Implications for clinical practice. American Psychologist, 62, 271-286.

Sue, D. W., Ivey, A.E., \& Pedersen, P. B. (1996) A Theory of Multicultural Counseling and Therapy, Belmont, CA, US: Thomson Brooks/Cole Publishing Co.

Tashakkori, A., \& Thompson, V. D. (1991). Race differences in self-perception and locus of control during adolescence and early adulthood. Genetic, Social, and General Psychology Monographs, 117, 135-152.

Thompson, C. P., Andersen, L. P., \& Bakeman, R. A. (2000). Effects of racial socialization and racial identity on acculturative stress in African American college students. Cultural Diversity and Ethnic Minority Psychology, 6, 196-210.

Toldson, I. A., \& Cooper, G. (2014). Historically Black colleges and universities data dashboard: Using the integrated postsecondary education data system to understand the current state of HBCUs. United States Department of Education, White House Initiative on Historically Black Colleges and Universities, 1-10.

Townsend, T. G., \& Belgrave, F. Z. (2000). The impact of personal identity and racial identity on drug attitudes and use among African American children. Journal of Black Psychology, $26,421-436$. 
Utsey, S. O., Ponterotto, J. G., Reynolds, A. L., \& Cancelli, A. A. (2000). Racial discrimination, coping, life satisfaction, and self-esteem among African Americans. Journal of Counseling \& Development, 78, 72-80.

Verkuyten, S. (2005) Ethnic group identification and group evaluation among minority and majority groups: Testing the multiculturalism hypothesis. Journal of Personality and Social Psychology, 88, 121-138.

Witherspoon, K. M., Speight, S. L., \& Thomas, A. J. (1997). Racial identity attitudes, school achievement, and academic self-efficacy among African American high school students. Journal of Black Psychology, 23, 344-357.

Worrell, F. C., \& Gardner-Kitt, D. L. (2006). The relationship between racial and ethnic identity in Black adolescents: The Cross racial identity scale and the multigroup ethnic identity measure. Identity: An International Journal of Theory \& Research, 6, 293-315.

Yee, M., \& Brown, R. (1992). Self-evaluation and intergroup attitudes in children aged three to nine. Child Development, 63, 619-62. 
Appendix A

Academic Self-Concept Scale (ASCS), (Reynolds et al., 1980)

Below is a list of statements addressing general feelings regarding the academic experience.

Respond to each item as it relates to you, personally. Base your ratings on how you feel most of the time. Use the following scale to rate each statement: SD (Strongly Disagree); D (Disagree); A (Agree); SA (Strongly Agree).

INDICATE YOUR RESPONSE BY CIRCLING THE APPROPRIATE LETTER(S). Be sure to answer all items. Please respond to each item independently (i.e., try not to be influenced by your previous choices.)

1. Being a student is a very rewarding experience.

$\begin{array}{llll}\text { SD } & \text { D } & \text { A } & \text { SA } \\ \text { SD } & \text { D } & \text { A } & \text { SA } \\ \text { SD } & \text { D } & \text { A } & \text { SA } \\ \text { SD } & \text { D } & \text { A } & \text { SA } \\ \text { SD } & \text { D } & \text { A } & \text { SA } \\ \text { SD } & \text { D } & \text { A } & \text { SA }\end{array}$

2. If I try hard enough, I will be able to get good grades.

3. Most of the time my efforts in school are rewarded.

4. No matter how hard I try I do not do well in school.

5. I often expect to do poorly on exams.

6. All in all, I feel I am a capable student.

7. I do well in my courses given the amount of time I dedicate to studying.

SD D A SA

8. My parents are not satisfied with my grades in college.

9. Others view me as intelligent.

SD D A SA

10. Most courses are very easy for me.

SD D A SA

11. I sometimes feel like dropping out of school.

SD D A $\quad$ SA

12. Most of my classmates do better in school than I do.

13. Most of my instructors think that I am a good student.

14. At times I feel college is too difficult for me.

SD $\quad \mathrm{D} \quad \mathrm{A} \quad \mathrm{SA}$

15. All in all, I am proud of my grades in college.

SD $\quad \mathrm{D} \quad \mathrm{A} \quad \mathrm{SA}$

16. Most of the time while taking a test I feel confident.

$\mathrm{SD}$

D A $\quad$ SA

17. I feel capable of helping others with their class work.

18. I feel teachers' standards are too high for me.

$\mathrm{SD}$

$\mathrm{D}$

A SA

SD D A $\quad$ SA

SD $\quad \mathrm{D} \quad \mathrm{A} \quad \mathrm{SA}$

19. It is hard for me to keep up with my class work.

SD $\quad \mathrm{D} \quad \mathrm{A} \quad \mathrm{SA}$

SD D A SA

20. I am satisfied with the class assignments that I turn in.

21. At times I feel like a failure.

SD

D

A SA

22. I feel I do not study enough before a test.

D A SA

23. Most exams are easy for me.

D A SA

24. I have doubts that I will do well in my major.

SD

25 . For me, studying hard pays off.

SD

SD

26. I have a hard time getting through school.

SD D

SD D

27. I am good at scheduling my study time.

SD $\quad \mathrm{D} \quad \mathrm{A} \quad \mathrm{SA}$

28. I have a fairly clear sense of my academic goals.

SD D

A $\quad$ SA

29. I'd like to be a much better student than I am now.

SD

$\mathrm{SD}$

30. I often get discouraged about school.

$\mathrm{SD}$

31. I enjoy doing my homework.

SD


32. I consider myself a very good student.

33. I usually get the grades I deserve in my courses.

34. I do not study as much as I should.

35 . I usually feel on top of my work by finals week.

36. Others consider me a good student.

37. I feel that I am better than the average college student.

38. In most of the courses, I feel that my classmates are better prepared than I am.

39. I feel that I do not have the necessary abilities for certain courses in my major.

40. I have poor study habits.

$\begin{array}{llll}\text { SD } & \text { D } & \text { A } & \text { SA } \\ \text { SD } & \text { D } & \text { A } & \text { SA } \\ \text { SD } & \text { D } & \text { A } & \text { SA } \\ \text { SD } & \text { D } & \text { A } & \text { SA } \\ \text { SD } & \text { D } & \text { A } & \text { SA } \\ \text { SD } & \text { D } & \text { A } & \text { SA } \\ & & & \\ \text { SD } & \text { D } & \text { A } & \text { SA } \\ & & & \\ \text { SD } & \text { D } & \text { A } & \text { SA } \\ \text { SD } & \text { D } & \text { A } & \text { SA }\end{array}$

Scoring: $\mathrm{SD}=1, \mathrm{D}=2, \mathrm{~A}=3, \mathrm{SA}=4$. Items $4,5,11,12,14,18,19,21,22,24,26,29,30,34,38$, 39 , and 40 are reversed scored, that is, $S D=4, D=3, A=2, S A=1$. Sum the scores for the 40 items; the higher the score, the higher the academic self-concept. 
Appendix B

Multigroup Ethnic Identity Measure (MEIM) (Phinney, 1992)

Below is a list of statements regarding ethnicity-related attitudes. Respond to each item as it relates to you, personally. Base your ratings on how you feel most of the time. Use the following scale to rate each statement: SD (Strongly Disagree); D (Disagree); A (Agree); SA (Strongly Agree).

INDICATE YOUR RESPONSE BY CIRCLING THE APPROPRIATE LETTER(S). Be sure to answer all items. Please respond to each item independently (i.e., try not to be influenced by your previous choices.)

1. I have spent time trying to find out more about my own ethnic group, such as its history, traditions, and customs.

SD D A SA

2. I am active in organizations or social groups that include mostly members of my own ethnic group. $\quad$ SD $\quad$ D $A$ SA

3.I have a clear sense of my ethnic background and what it means to me.

$$
\text { SD D A SA }
$$

4.I like meeting and getting to know people from ethnic groups other than my own.

$$
\text { SD D A SA }
$$

5.I think a lot about how my life will be affected by my ethnic group membership.

$$
\text { SD D A SA }
$$

6.I am happy that I am a member of the group I belong to.

$$
\text { SD D A SA }
$$

7.I sometimes feel it would be better if different ethnic groups did not try to mix together.

$$
\text { SD D A SA }
$$

8.I am not very clear about the role of my ethnicity in my life.

$$
\text { SD D A SA }
$$

9.I often spend time with people from ethnic groups other than my own.

$$
\text { SD D A SA }
$$

10.I really have not spent much time trying to learn about the culture and history of my ethnic group. SD D A $\quad$ SA

11.I have a strong sense of belonging to my own ethnic group.

$$
\text { SD D A SA }
$$

12. I understand pretty well what my ethnic group membership means to me, in terms of how I relate to my own group and other groups.

$$
\text { SD D A SA }
$$

13. To learn more about my ethnic background, I have often talked to other people about $\begin{array}{lllll}\text { my ethnic background. } & \text { SD } & \text { D } & \text { A }\end{array}$

14.I have pride in my ethnic group and its accomplishments. 
15. I do not try to become friends with people from other ethnic groups.

$$
\text { SD D A SA }
$$

16. I participate in cultural practices of my own group, such as special food music or customs.

$$
\text { SD D A SA }
$$

17. I am involved in activities with people of other ethnic groups.

$$
\text { SD D A SA }
$$

18. I feel a strong attachment toward my ethnic group.

$$
\text { SD D A SA }
$$

19.I enjoy being around people of ethnic groups other than my own.

$$
\text { SD D A SA }
$$

20.I feel good about my culture or ethnic background.

$$
\text { SD D A SA }
$$

Scoring: $\mathrm{SD}=1, \mathrm{D}=2, \mathrm{~A}=3, \mathrm{SA}=4$. Items $7,8,10$, and 15 are reversed scored, that is, $\mathrm{SD}=4, \mathrm{D}=3$, $\mathrm{A}=2, \mathrm{SA}=1$. Sum the scores for the 20 items; the higher the score, the more achieved the ethnicidentity.

*Note: The Ethnic Identity Scale, or Factor I score, is based on 14 of the 20 MEIM items (i.e., items 1, 2, 3, 5, 6, 8, 10, 11, 12, 13, 14, 16, 18, \& 20). Factor II, the Other Group Orientation (OGO) score is obtained from the remaining 6 items (i.e., items 4, 7, 9, 15, 17, \& 19). 
Appendix C

Rosenberg Self-Esteem Scale (RSES) (Rosenberg, 1965)

Below is a list of statements dealing with your general feelings about yourself. If you strongly agree, circle SA. If you agree with the statement, circle A. If you disagree, circle D. If you strongly disagree, circle SD.

1. On the whole, I am satisfied with myself.

SA A D $\quad$ SD

2. At times, I think I am no good at all.

SA A D $\quad$ SD

3. I feel that I have a number of good qualities.

SA A D $\quad$ SD

4. I am able to do things as well as most other people.

SA A D SD

5. I feel I do not have much to be proud of.

SA A $\quad$ D $\quad$ SD

6. I certainly feel useless at times.

SA A

D SD

7. I feel that I'm a person of worth, at least on an equal plane with others.

SA A D $\quad$ SD

8. I wish I could have more respect for myself.

SA A D $\quad$ SD

9. All in all, I am inclined to feel that I am a failure.

SA A $\quad$ D $\quad$ SD

10. I take a positive attitude toward myself.

SA A D $\quad$ SD

Scoring: $\mathrm{SA}=3, \mathrm{~A}=2, \mathrm{D}=1, \mathrm{SD}=0$. Items 2, 5, 6, 8, and 9 are reversed scored. Sum the scores for the 10 items; the higher the score, the higher the self-esteem. 
Appendix D

Marlowe-Crowne Social Desirability Scale: Short Form C (MC-C) (Crowne \& Marlowe, 1960; Reynolds, 1982)

Listed below are a number of statements concerning personal attitudes and traits. Read each item and decide whether the statement is True or False as it pertains to you personally.

1. It is sometimes hard to go on with my work if I am not encouraged.

2. I sometimes feel resentful when I don't get my way.

T F

3. On a few occasions, I have given up doing something because I thought too little of my ability.

4. There have been times when I felt like rebelling against people in authority even when I knew they were right.

5. No matter who I'm talking to, I'm always a good listener.

6. There have been occasions when I took advantage of someone.

7. I'm always willing to admit it when I make a mistake.

8. I sometimes try to get even rather than to forgive and forget.

9. I am always courteous, even to people who are disagreeable.

10. I have never been irked when people expressed ideas very different from my own.

11. There have been times when I was quite jealous of the good fortune of others.

12. I am sometimes irritated by people who ask favors of me.

13. I have never deliberately said something that hurt someone's feelings. $\quad T \quad F$

Note: Items given a score when True: 5, 7, 9, 10, and 13. Items given a score when False: 1, 2, 3, $4,6,8,11$, and 12. Sum the scores for the 13 items; the higher the score, the higher the socialdesirability. 
Appendix E

\section{Demographic Questionnaire}

The purpose of this questionnaire is for you to provide some basic background information about yourself and your academic experience. Please answer the following questions.

1. What is your sex:

Female Male

2. What is your identified race/ethnicity:

__ Black or African-American __ White __ Hispanic or Latino(a)

_ Native American or American Indian Asian or Pacific Islander

_ Other

3. What is your age:
18-21 22-25 26-30 31-40 41 and over

4. What is your current class year:

_ Freshman __ Sophomore __ Junior __ Senior ___ Graduate/Professional

5. What is your current or intended major?

6. What is your cumulative GPA*:
4.0-3.5 3.5-3.0 3.0-2.5 2.5-2.0 2.0 and under

*GPA Range Translation Scale
4.0-3.5 = 4
3.5-3.0 $=3$
3.0-2.5 $=2$
2.5-2.0 $=1$
2.0 and under $=0$ 
Appendix F

\section{Recruitment Letter}

\section{Dear Participant,}

This letter is a request for you to take part in a research project to explore the relation of ethnic identity to self-esteem and academic self-concept of African American students at WVU. This dissertation project is being conducted by Drummond Bush, MA in the Department of Counseling, Rehabilitation Counseling and Counseling Psychology at WVU with supervision of Dr. Jeffrey Daniels, an associate professor in the College of Education and Human Services, for a Doctoral Degree in Counseling Psychology. Your participation in this project is greatly appreciated and will take approximately 10 to 15 minutes to fill out the surveys and questionnaire. Upon completion of the survey, if you choose, you will be asked to enter a preferred e-mail address in order to become eligible for 1 of 10, \$20.00 Amazon.com gift cards. The winners will be chosen by random drawing and gift cards will be sent via the e-mail address provided. If you do not provide an e-mail, you will not be entered into the prize drawing.

Your involvement in this project will be kept as confidential as legally possible. All data will be reported in the aggregate. Eligible participants must be at least 18-years-old, self-identify as African American or Black (either solely or as a part of their race/ethnicity), and be currently enrolled in an on-campus degree program. I will not ask any information that should lead back to your identity as a participant. Your participation is completely voluntary. You may skip any question that you do not wish to answer and you may discontinue at any time. Your class standing will not be affected if you decide either not to participate or to withdraw. West Virginia University's Institutional Review Board acknowledgment of this project is on file.

I hope that you will participate in this research project, as it could be beneficial in understanding the influence of ethnic identity on the college experience for African American students at WVU. Thank you very much for your time. Should you have any questions about this letter or the research project, please feel free to contact Drummond Bush, MA at (817)307-1468 or by email at dbush5@mix.wvu.edu or Dr. Jeff Daniels at (304) 293-2235 or by e-mail at Jeffrey.Daniels@mail.wvu.edu.

Please copy and paste the following URL into your web browser to take the survey: https://wvuhre.az1.qualtrics.com/SE/?SID=SV 9t6bYS2wIW8bEH3

Thank you for your time and help with this project.

Sincerely,

Drummond Bush, MA

Jeff Daniels, Ph.D. 
Appendix G

\section{Demographic Summary of Participants $(n=91)$}

1. Sex: $\quad \underline{\text { Female }}=\mathbf{5 9}(65 \%)$

Male $=\mathbf{2 3}(25 \%)$

$\underline{\text { Undisclosed }}=\mathbf{9}(10 \%)$

2. Identified Race/Ethnicity: Black/African American (Singular Ethnicity) $=\mathbf{7 7}(85 \%)$

Other (Multiple Ethnicity includes Black/AA) $=\mathbf{1 0}(11 \%)$

Native American (Multiple Ethnicity includes Black/AA) $=\mathbf{1}(1 \%)$

White (Multiple Ethnicity includes Black/AA) $=3$ (3\%)

3. Age: $\underline{18-21}=\mathbf{4 8}(53 \%)$

$\underline{22-25}=\mathbf{2 4}(26 \%)$

$\underline{26-30}=\mathbf{9}(10 \%)$

$\underline{31-40}=\mathbf{6}(7 \%)$

$\underline{41+}=\mathbf{4}(4 \%)$

4. Class Year: $\underline{\text { Freshman }}=\mathbf{1 9}(21 \%)$

$\underline{\text { Sophomore }}=\mathbf{9}(10 \%)$

$\underline{\text { Junior }}=\mathbf{1 5}(16 \%)$

$\underline{\text { Senior }}=\mathbf{1 8}(20 \%)$

Graduate/Professional $=\mathbf{3 0}(33 \%)$

5. Current or Intended Major: (See List Below)

Graduate/Professional $(\boldsymbol{n}=\mathbf{3 0}) * 20$ unique majors

Kinesiology

Animal Physiology

Social Work

Dietetics

Elementary Education

Chemistry

Counseling

English

Agriculture \& Resource Economics

Biomedical Science

Journalism

Epidemiology

$\underline{\text { Child Development \& Family Studies }}=\mathbf{2}$

$\underline{\text { Business }}=\mathbf{3}$

Integrated Marketing Communications $=\mathbf{3}$

Law

Medicine

Pharmacy $=\mathbf{2}$

Counseling/Clinical Psychology $=\mathbf{3}$

$\underline{\text { Graduate }(\text { Undefined })}=\mathbf{3}$ 


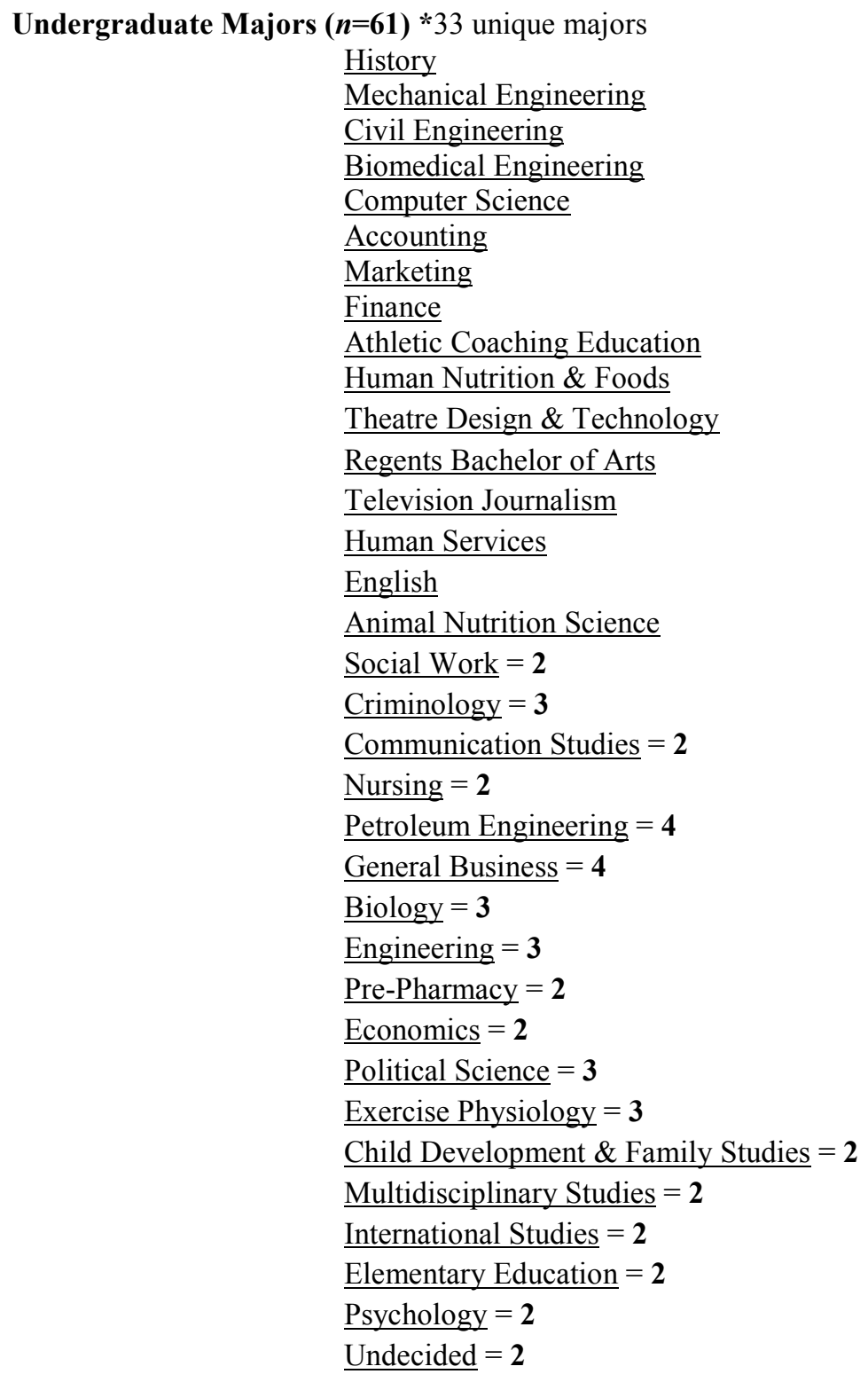

\section{Cumulative GPA:}

$$
\begin{aligned}
& \frac{4.0-3.5}{3.5}=\mathbf{2 8}(31 \%) \\
& \frac{3.0}{3.0}-2.5=\mathbf{3 3}(36 \%) \\
& \frac{20}{2.5}-2.0=5(22 \%) \\
& \underline{2.0} \geq=1(1 \%) \\
& \underline{\mathrm{NA}}=\mathbf{4}(4 \%)
\end{aligned}
$$

